\title{
Lyapunov Exponents of Control Flows ${ }^{1}$
}

\author{
Fritz Colonius, Universität AUgSBURG \\ WolfGang Kliemann, Iowa State University
}

\begin{abstract}
The use of Lyapunov exponents in the theory of dynamical systems or stochastic systems is often based on Oseledeč's Multiplicative Ergodic Theorem. For control systems this is not possible, because each (sufficiently rich) control system contains dynamics that are not Lyapunov regular. In this paper we present an approach to study the Lyapunov spectrum of a nonlinear control system via ergodic theory of the associated control flow and its linearization. In particular, it turns out that all Lyapunov exponents are attained over so called chain control sets, and they are integrals of Lyapunov exponents on control sets with respect to flow invariant measures, whose support is contained in the lifts of control sets to $U \times M$, where $U$ is the space of admissible control functions and $M$ is the state space of the system. For the linearization of control systems about rest points the extremal Lyapunov exponents are analyzed, which leads to precise criteria for the stabilization and destabilization of bilinear control systems, and to robustness results for linear systems. The last section is devoted to a nonlinear example, where we combine the analysis of the global controllability structure with local linearization results and Lyapunov exponents to obtain a complete picture of control, stabilization and robustness of the system.
\end{abstract}

Keywords. nonlinear control systems, dynamical systems, control sets, chain control sets, linearization, ergodic theory, stabilization, robustness

1980 Mathematics subject classifications: $93 \mathrm{C} 10,58 \mathrm{~F} 10,58 \mathrm{~F} 11,93 \mathrm{~B} 05,93 \mathrm{D} 20$

\section{Introduction}

The local study of ordinary differential equations and smooth dynamical systems via linearization techniques and Lyapunov exponents goes back to Lyapunov (1892) and is exemplified e.g. in the books by Nemytskii and Stepanov (1960), Cesari (1971), Hahn (1967), Mañé (1987) and many others. In the time dependent case, Oseledeč's multiplicative ergodic theorem (1968) shows, how to obtain results with probability one about Lyapunov regular points, i.e. about Lyapunov exponents, invariant manifolds, exponential stability, and behavior under small perturbations. Likewise, entropy theory, bifurcation theory, strange attractors etc. can be closely related to Lyapunov exponents of dynamical systems. While Oseledeč's theorem is also a convenient starting point for the local analysis of nonlinear stochastic systems in the form of stochastic flows (compare e.g. many contributions in this volume), this is not the case for control systems.

Consider a control system of the form

$$
\dot{x}(t)=X_{0}(x(t))+\sum_{i=1}^{m} u_{i}(t) X_{i}(x(t)), \quad x(0)=x_{0} \in M, \quad t \in \mathbf{R}
$$

\footnotetext{
${ }^{1}$ Research supported in part by NSF grant no. DMS 8813976 and DFG grants no. Co124/6-1 and Co124/8-1.
} 
on a finite dimensional, smooth manifold $M$ with smooth vectorfields $X_{0}, \ldots, X_{m}$. The $u_{i}(\cdot)$ are the admissible control functions in $\mathcal{U}=\left\{u: \mathbf{R} \rightarrow U \subset \mathbf{R}^{m}\right.$, locally integrable $\}$.

Typical questions in control theory include: Given two points $y, z \in M$, does there exist a control function $u \in \mathcal{U}$ such that $y=x(t, z, u)$ ? Does there exist a control $u \in \mathcal{U}$ such that the system can be stabilized at a given trajectory, e.g. a rest point? Is this stabilization robust with respect to small (or large) perturbations of the vector fields? If the trajectory of $\dot{x}(t)=X_{0}(x(t))$ has a complicated behavior, does there exist a $u \in \mathcal{U}$ such that the corresponding solution of (1.1) has a simple (desired) behavior? Can these goals be achieved via feedback controls, i.e. $u=f(x)$ ? Compare e.g. Wonham (1979), Isidori (1989), or Nijmeijer and van der Schaft (1990) for a range of control theoretic topics.

Many techniques in control theory are global in nature, compare the literature mentioned above. If linearization techniques are used (e.g. around rest points for all $X_{0} \ldots X_{m}$ ), then the system is usually linearized with respect to $x$ and $u$, yielding a linear control system of the form

$$
\dot{x}(t)=A x(t)+B u(t)
$$

But the typical problems listed above show that linearization with respect to $u$ is often not appropriate, because one looks for the existence of some $u \in \mathcal{U}$ with a desired property, which is a global question in $u$. On the other hand, linearization with respect to $x$ around a rest point leads to a bilinear control system

$$
\dot{x}(t)=A_{0} x(t)+\sum_{i=1}^{m} u_{i}(t) A_{i} x(t)
$$

Since control systems contain matrix functions that are not Lyapunov regular, one cannot use Oseledeć's theorem or similar approaches to describe the Lyapunov spectrum of (1.3) and its implications for the local behavior of the nonlinear system. Other spectral concepts, like the dynamical spectrum (compare e.g. Sacker and Sell (1978) and Johnson et al. (1987)) or the Morse spectrum based on Morse decompositions of projected, linearized flows (compare Sections 3. and 4.) are too crude to describe the local behavior of (1.1) appropriately.

In this paper we present an approach to the study of global and local open loop properties of control systems, which is based on the concept of control flows on $\mathcal{U} \times M$ for the global ideas, and on the induced flows on $\mathcal{U} \times T M$ and $\mathcal{U} \times \mathrm{P} M$ for local analysis. (Here $T M$ and $P M$ are the tangent and the projective bundle over $M$.) It turns out that a careful study of the control properties of the systems on $M$ and on $P M$, using control and chain control sets, and the approximation of spectral values via Lyapunov exponents of regular elements (using ergodic theory or approximation by piecewise constant, periodic solutions) allows the characterization of Lyapunov exponents for (topologically) "thick" sets in $\mathcal{U} \times M$. Our techniques are a combination of methods from the theory of dynamical systems and from control theory, with many of them being inspired by ideas from the theory of stochastic flows.

Our goal is to develop techniques to obtain exact criteria for nonlinear control systems with respect to the problems mentioned above. For linear systems of the form (1.2) 
with $U=\mathbf{R}^{m}$ methods from linear algebra together with Lyapunov functions, Riccati equations etc. can be used to solve this problem, and the explicit constructions yield feedback controls via gain matrices automatically. But if $U \subset \mathbf{R}^{m}$ is bounded, then the problem becomes 'nonlinear' (compare Section 5.) and many techniques break down, i.e. yield only sufficient conditions. For nonlinear systems, Lyapunov function techniques still work for some problems (compare e.g. Sontag (1983)). Other authors have developed approaches to stabilization using center manifolds of reference systems (see e.g. Byrnes and Isidori (1989), Knobloch (1988)), and the feedback problem is treated e.g. in Sussmann (1979) and Sontag (1989). The approach presented here uses control flows and Lyapunov exponents as a unifying tool.

In Section 2. we describe the set up and the notations used throughout this paper, in particular control flows on $\mathcal{U} \times M$, linearized flows on $\mathcal{U} \times T M$ and their projections to $\mathcal{U} \times \mathbf{P} M$ are introduced. Since our results rely on some previous work of the authors, we review (with minor extensions) in Section 3. control sets, chain control sets and their characterizations for the flows presented before. Section 4 introduces Lyapunov exponents for (1.1) and characterizes the Lyapunov spectrum over control and chain control sets in terms of ergodic theory for the associated flows. In Section 5 we demonstrate for the problems of stabilization of bilinear systems and for robustness of linear systems that the Lyapunov exponent approach does yield exact criteria for control systems (and also for classes of stochastically perturbed systems). Finally in Section 6 . the simple nonlinear, controlled Verhulst equation is treated with respect to controllability, stabilization and robustness, using the results from Sections 3.-5. The approach developed there can be used for general nonlinear control systems with one-dimensional state space.

\section{Control Systems, Control Flows, and their Linearizations}

In this section we set up the basic concepts and notations, which will be used throughout the paper. We will consider the following class of nonlinear control systems on a para-compact connected $d$-dimensional Riemannian $C^{\infty}$ manifold $M$

$$
\dot{x}(t)=X_{0}(x(t))+\sum_{i=1}^{m} u_{i}(t) X_{i}(x(t)), \quad t \in \mathbf{R}, \quad x(0)=x_{0} \in M
$$

where the $X_{0}, \ldots, X_{m}$ are $C^{\infty}$ vectorfields and the admissible controls are $u:=\left(u_{i}\right) \in$ $\mathcal{U}:=\{u: \mathbf{R} \rightarrow U$, locally integrable $\}$ with $U \subset \mathbf{R}^{m}$ compact and convex. We assume that for all $u \in \mathcal{U}$ and $x_{0} \in M$ the equation (2.1) has a unique solution $\varphi\left(\cdot, x_{0}, u\right)$, defined on $\mathbf{R}$ with $\varphi\left(0, x_{0}, u\right)=x_{0}$. Although most of our results remain true (with appropriate modifications) for more general systems of the type $\dot{x}(t)=X(x(t), u(t))$, (2.1) is a particularly nice and popular class of nonlinear control systems, because the controls appear linearly, compare e.g. Isidori (1989) and Nijmeijer and van der Schaft (1990) for basic concepts and results in nonlinear systems theory.

Because the admissible control functions in (2.1) are time dependent, for each $u \in \mathcal{U}$ the vector field on the right hand side is time dependent, and thus a flow for these 
systems has to be constructed over the space $\mathcal{U} \times M$. This can be done as follows: On $\mathcal{U}$ consider the natural shift defined by

$$
\theta: \mathbf{R} \times \mathcal{U} \rightarrow \mathcal{U}, \quad \theta_{t} u(\cdot)=u(t+\cdot)
$$

Then $\theta$ defines a continuous dynamical system on $\mathcal{U}$, if we equip $\mathcal{U}$ with the weak* topology of $L^{\infty}\left(\mathbf{R}, \mathbf{R}^{m}\right)=\left(L^{1}\left(\mathbf{R}, \mathbf{R}^{m}\right)\right)^{*}$. $\mathcal{U}$ with this topology is compact and metrizable. Note that the weak* topology on $\mathcal{U}$ is well suited for the study of (2.1), because convergence of $u_{n} \rightarrow u$ in $\mathcal{U}$ implies uniform convergence of $\varphi\left(\cdot, x_{0}, u_{n}\right) \rightarrow \varphi\left(\cdot, x_{0}, u\right)$ in $M$ on compact time intervals, compare Colonius and Kliemann $\left(1990^{a}\right)$ for these facts and a more detailed study of the shift space $(\mathcal{U}, \theta)$.

To the control system (2.1) we associate a control flow in the following manner:

$$
\phi: \mathbf{R} \times \mathcal{U} \times M \rightarrow \mathcal{U} \times M, \quad \phi_{t}(u, x)=\left(\theta_{t} u, \varphi(t, x, u)\right),
$$

where $\theta_{t}$ is the shift on $\mathcal{U}$, and $\varphi(\cdot, x, u)$ denotes the solution of equation (2.1) as above. $\phi$ is a skew product flow on $\mathcal{U} \times M$, because $\phi_{t+s}=\phi_{t} \circ \phi_{s}$, and $\varphi(t+s, x, u)=$ $\varphi\left(t, \varphi(s, x, u), \theta_{s} u\right)$. In fact, $\phi$ defines a continuous dynamical system on $\mathcal{U} \times M$, compare Lemma 3.4 in Colonius and Kliemann $\left(1990^{\circ}\right)$.

If $\mathcal{U}$ carries a $\theta$-invariant probability measure, then (2.3) can be interpreted as a stochastic flow, see Colonius and Kliemann $\left(1990^{\circ}\right)$ for the set up and some results on ergodic theory of control and stochastic flows, which can be proved in this unifying framework.

In this paper we are interested in linearization techniques for nonlinear control systems. Linearizing the system (2.1) with respect to the state variable $x$, we obtain a system defined on the tangent bundle $T M$ :

$$
\begin{aligned}
& (\dot{T} x)(t)=T X_{0}(T x)+\sum_{i=1}^{m} u_{i}(t) T X_{i}(T x), \quad t \in \mathbf{R}, \\
& (T x)(0)=\left(x_{0}, v_{0}\right) \in T_{x_{0}} M, \text { the tangent space at } x_{0} \in M
\end{aligned}
$$

where for a smooth vectorfield $X$ on $M$ its linearization is denoted by $T X=(X, D X)$. Locally this means: If $X_{j}=\sum_{k=1}^{d} \alpha_{k_{j}}(x) \frac{\partial}{\partial x_{k}}$, denote the Jacobian of the coefficient functions by $A_{j}(x)=\left(\frac{\partial \alpha_{k_{j}}(x)}{\partial x_{e}}\right)$. Then $T X_{j}(x, v)=\left(\alpha_{j}(x), A_{j}(x) v\right)$, and (2.4) is a pair of coupled differential equations, given locally by

$$
\begin{aligned}
& \dot{x}(t)=\alpha_{0}(x)+\sum_{i=1}^{m} u_{i}(t) \alpha_{i}(x), \quad x(0)=x_{0}, \\
& \dot{v}(t)=A_{0}(x) v+\sum_{i=1}^{m} u_{i}(t) A_{i}(x) v, \quad v(0)=v_{0} .
\end{aligned}
$$

Note that we have linearized (2.1) only with respect to the state $x \in M$, and not with respect to the control $u \in U$. (Linearization with respect to $x$ and $u$ is common practice in the control theory literature, but we are interested in results that are global in $u \in \mathcal{U}$.) 
In particular, if $x(t) \equiv x_{0} \in M$ is a rest point for each vectorfield $X_{i}, i=0, \ldots, m$, then the linearized equation is a bilinear control system. (Linearization with respect to $x$ and $u$ leads to linear control systems, which for unbounded control values $u \in \mathbf{R}^{m}$ can be treated by methods from linear algebra.)

The system (2.4) induces a control system on the projective bundle $\mathrm{P} M$, given by

$$
\begin{aligned}
(\dot{\mathbf{P}} x)(t) & =\mathbf{P} X_{0}(\mathbf{P} x)+\sum_{i=1}^{m} u_{i}(t) \mathbf{P} X_{i}(\mathbf{P} x), \quad t \in \mathbf{R}, \\
(\mathbf{P} x)(0) & =\left(x_{0}, s_{0}\right) \in \mathbf{P}_{x_{0}} M, \text { the projective space at } x_{0} \in M,
\end{aligned}
$$

where $\mathrm{P} X$ is the projection of a vector field $T X$ on $T M$ onto $\mathrm{P} M$, i.e. the $\mathrm{P} X_{j}$ read locally

$$
\mathrm{P} X_{j}(x, s)=\left(\alpha_{j}(x), h\left(A_{j}(x), s\right)\right), \quad h\left(A_{j}(x), s\right)=\left[A_{j}(x)-s^{T} A_{j}(x) s \cdot i d\right] s .
$$

Here ${ }^{T}$ denote transposition, and $i d$ is the $(d \times d)$ identity matrix. The trajectories of the control system (2.4) will be denoted by $T \varphi(\cdot, T x, u)$, and those of $(2.6)$ by $\mathbf{P} \varphi(\cdot, \mathbf{P} x, u)$.

Note that using the same construction, it is also possible to lift the system (2.1) to $F M$, the flag manifold over $M, G \ell(M)$, the frame bundle over $M$, or $\mathcal{O}(M)$, the orthonormal frame bundle over $M$ (see e.g. San Martin and Arnold (1986) or San Martin (1986)), but we will not make explicit use of these systems in this paper.

Just as the underlying control system (2.1), the linearized system (2.4) on $T M$ and the projected system (2.6) on PM give rise to associated flows

$$
\begin{array}{ll}
T \phi: \mathbf{R} \times \mathcal{U} \times T M \rightarrow \mathcal{U} \times T M, & T \phi_{t}(u, T x)=\left(\theta_{t} u, T \varphi(t, T x, u)\right), \\
\mathbf{P} \phi: \mathbf{R} \times \mathcal{U} \times \mathbf{P} M \rightarrow \mathcal{U} \times \mathbf{P} M, & \mathbf{P} \phi_{t}(u, \mathbf{P} x)=\left(\theta_{t} u, \mathbf{P} \varphi(t, \mathbf{P} x, u)\right),
\end{array}
$$

which are also skew product flows, defining continuous dynamical systems on $\mathcal{U} \times T M$ (and $\mathcal{U} \times \mathbf{P} M$, respectively). In this paper we will be concerned mainly with the Lyapunov exponents of (2.8) and their characterization via (2.9). This analysis will require some characterizations of the flows $T \phi$ and $\boldsymbol{P} \phi$ in terms of concepts from the theory of dynamical systems. These results are presented in the next section.

\section{Control Sets, Chain Control Sets, and Subbundle Decompositions}

Consider again the underlying control system on the $d$-dimensional manifold $M$

$$
\dot{x}(t)=X_{0}(x(t))+\sum_{i=1}^{m} u_{i}(t) X_{i}(x(t)), \quad t \in \mathbf{R}, \quad x(0)=x_{0} \in M,
$$

with $u \in \mathcal{U}$. In order to avoid degenerate situations, we will assume throughout the rest of this paper the following integrability condition

$$
\operatorname{dim} \mathcal{L} \mathcal{A}\left\{X_{0}+\sum u_{i} X_{i} ;\left(u_{i}\right) \in U\right\}(x)=d \quad \text { for all } x \in M,
$$


where for a set $\mathcal{X}$ of vectorfields on $M, \mathcal{L} \mathcal{A}\{\mathcal{X}\}$ denotes the lie algebra generated by $\mathcal{X}$. Associated with (2.1) are the positive semigroup $\mathcal{S}^{+}$, the negative semigroup $\mathcal{S}^{-}$, and the group $\mathcal{G}$ of solution diffeomorphisms generated by piecewise constant controls: Denote by $N:=\left\{X_{0}+\sum u_{i} X_{i} ;\left(u_{i}\right) \in U\right\}$ the admissible (time independent) right hand sides of (2.1), and define

$$
\begin{aligned}
\mathcal{S}^{+} & =\left\{\exp \left(t_{n} Y_{n}\right) \circ \cdots \circ \exp \left(t_{1} Y_{1}\right) ; Y_{i} \in N, t_{i} \geq 0, i=1, \ldots, n \in \mathrm{N}\right\} \\
\mathcal{S}^{-} & =\left\{\exp \left(t_{n} Y_{n}\right) \circ \cdots \circ \exp \left(t_{1} Y_{1}\right) ; Y_{i} \in N, t_{i} \leq 0, i=1, \ldots, n \in \mathrm{N}\right\} \\
\mathcal{G} & =\left\{\exp \left(t_{n} Y_{n}\right) \circ \cdots \circ \exp \left(t_{1} Y_{1}\right) ; Y_{i} \in N, t_{i} \in \mathrm{R}, i=1, \ldots, n \in \mathrm{N}\right\}
\end{aligned}
$$

The subsets of $\mathcal{S}^{+}, \mathcal{S}^{-}, \mathcal{G}$ with $\sum_{i=1}^{n}\left|t_{i}\right| \leq t$ are denoted by $\mathcal{S}_{\leq t}^{+}, \mathcal{S}_{\leq t}^{-}, \mathcal{G}_{\leq t}$, and similarly for $\sum\left|t_{i}\right|=t$ by $\mathcal{S}_{t}^{+}, \mathcal{S}_{t}^{-}, \mathcal{G}_{t}$. Under Hypothesis $(\mathrm{H})$ we have for the system (2.1):

\subsection{LEMMA.}

(i) $\mathcal{G}$ acts transitively on $M$, i.e. $\mathcal{G} x=M$ for all $x \in M$.

(ii) int $\mathcal{S}_{\leq t}^{+} x \neq \phi$, and int $\mathcal{S}_{\leq t}^{-} x \neq \phi$ for all $t>0$, i.e. the reachable set up to time $t>0$ (and the controllable set up to time $-t<0$ ) has nonvoid interior on $M$ for all $x \in M$. This property is often called 'local accessibility' in the control theory literature.

(iii) The (positive) orbits up to time $t>0$ on $M$, defined by

$$
\mathcal{O}_{\leq t}^{+}(x)=\{y \in M ; \text { there exist } u \in \mathcal{U} \text { and } 0 \leq s \leq t \text { with } y=\varphi(s, x, u)\}
$$

have nonvoid interior in $M$, and $\overline{\mathcal{S}_{\leq t}^{+} x}=\overline{\mathcal{O}_{\leq t}^{+}(x)}$ for all $t>0$, all $x \in M$. Similarly for the negative orbits $\mathcal{O}_{\leq t}^{-}(x)$.

For a proof see e.g. Isidori (1989) and Nijmeijer and van der Schaft (1990).

Lemma 3.1 shows that under integrability of $N$ the manifold $M$ is the state space of appropriate dimension for the system (2.1), if $(H)$ holds. This does not imply, however, that (2.1) is completely controllable on $M$. We define the subsets, in which (2.1) is controllable, as follows:

3.2. Definition: A set $D \subset M$ is called a control set of (2.1), if

(i) for every $x \in D$ there exists $u \in \mathcal{U}$ such that $\varphi(t, x, u) \subset D$ for all $t \geq 0$,

(ii) for all $x \in D$ we have $\overline{\mathcal{O}^{+}(x)} \supset D$,

(iii) $D$ is maximal with respect to the properties (i) and (ii).

If, furthermore, $\bar{D}=\overline{\mathcal{O}+(x)}$ for all $x \in D$, then $D$ is called an invariant control set.

We are, in particular, interested in control sets with nonvoid interior, and for these sets the following results hold: 


\subsection{LEMMA.}

(i) Let $D \subset M$ be a control set with int $D \neq \phi$. Then

(a) for all $x \in D, y \in$ int $D$ there exist $t \geq 0$ and $g \in \mathcal{S}_{\leq t}^{+}$such that $y=g x$,

(b) $\overline{\text { int } D}=\bar{D}$.

(ii) (a) Control sets are pairwise disjoint, and in general neither open nor closed. A control set with nonvoid interior is closed iff it is invariant.

(b) If $M$ is compact, then there exist at least one invariant and one open control set in $M$, the invariant control sets have nonvoid interior, i.e. are closed.

A proof of these facts can be found e.g. in Colonius and Kliemann (1989).

The control sets of (2.1) are ordered in the following way: Let $D_{1}$ and $D_{2}$ be control sets, then we define

$$
D_{1} \prec D_{2} \text { if there exist } x \in D_{1} \text { and } y \in D_{2} \text { with } y \in \overline{\mathcal{O}^{+}(x)} \text {. }
$$

The relation (3.2) defines an order on the control sets, where the closed (i.e. invariant) control sets are maximal elements of $\prec$, the open control sets are minimal elements. If $M$ is compact, then the maximal (minimal) elements are exactly the closed (open) control sets, compare Colonius and Kliemann (1990'), Lemma 3.11.

For this paper, we are interested in control sets (with nonvoid interior) mainly for three reasons:

(i) Control sets $D \subset M$ can be lifted to invariant sets $\mathcal{D} \subset \mathcal{U} \times M$, and the flow $\phi$, defined in (2.3), restricted to $\mathcal{D}$ is a dynamical system with several interesting properties.

(ii) Over the control sets $D \subset M$, the control structure of the system on the projective bundle PM (compare (2.6)) can be described, which allows us to give a characterization of the Lyapunov exponents.

(iii) The control sets $\mathbf{p} D \subset \mathbf{P} M$ of (2.6) can be lifted to $\mathcal{U} \times \mathbf{P} M$, where an ergodic theory for Lyapunov exponents can be developed.

As a next step, we will therefore study the lifts of control sets and the control structure of (2.6).

For a control set $D \subset M$, with int $D \neq \phi$, we define its lift to $\mathcal{U} \times M$ by

$$
\mathcal{D}=c \ell\{(u, x) \in \mathcal{U} \times M ; \varphi(t, x, u) \in \operatorname{int} D \text { for all } t \in \mathbf{R}\}
$$

where the closure is taken with respect to the weak* topology in $\mathcal{U}$ and the given topology on $M$. Since $\mathcal{D}$ is a closed, $\phi$-invariant set, we can consider the dynamical system $\left(\mathcal{D},\left.\phi\right|_{\mathcal{D}}\right)$. Using this flow, control properties of (2.1) can be described in terms of concepts from the theory of dynamical systems, such as topological mixing, topological transitivity, chaos, compare Colonius and Kliemann $\left(1990^{a}\right)$, Section 3.

In order to analyze the linearized systems (2.4) and (2.6), we will need a condition similar to (H) for the system on PM:

$$
\operatorname{dim} \mathcal{L} \mathcal{A}\left\{\mathbf{P} X_{0}+\sum u_{i} \mathbf{P} X_{i} ;\left(u_{i}\right) \in U\right\}(x, v)=2 d-1 \text { for all }(x, v) \in \mathbf{P} M .
$$


Compare San Martin and Arnold (1986) for a detailed discussion of Assumption (PH) and its consequences.

3.4. REMARK: If $C \subset M$ is an invariant control set of (2.1), then, under (H), int $C \neq \phi$. Hence, under $(\mathrm{PH})$, all the consequences of the Lemmas 3.1 and 3.3 hold for the control sets of (2.6) on the state space Pint $C$, the projective bundle over the manifold int $C \subset M$. In particular, if the system (2.1) is completely controllable on $M$, then $C=\operatorname{int} C=M$, and we can consider the system on PM. This is the case studied by San Martin and Arnold (1986) and by San Martin (1986) in a stochastic context.

The following theorem is a slight generalization of Theorem 3.10 in Colonius and Kliemann $\left(1990^{b}\right)$, using the ideas in San Martin and Arnold (1986) and San Martin (1986). It shows that over the invariant control sets of (2.1) the control structure of (2.6) is uniform:

3.5. Theorem. Assume that (PH) holds, and let $C$ be a compact invariant control set of (2.1). Then it holds for the projected system (2.6) on Pint $C$ :

(i) There are $k$ control sets $\mathbf{p} D_{i}$ with nonvoid interior in $\operatorname{Pint} C$ and $1 \leq k \leq d$; we call these the main control sets of the system.

(ii) The main control sets are linearly ordered, where the order is defined as in (3.2), where $\mathcal{O}^{+}$now denotes the positive orbit of (2.6). We enumerate the sets such that $\mathbf{p}_{1} D_{1} \prec_{\mathbf{p}} D_{2} \prec_{\mathbf{p}} \cdots \prec_{\mathbf{p}} \mathbf{p} D_{k}$. In particular, $\mathbf{p}_{\mathbf{p}} D_{k}$ is the unique invariant control set over $C$.

A more explicit description of the control sets $\mathbf{p} D_{i}$ is given in Section 5 . for systems linearized around a rest point.

Now consider the linearized system (2.4) on the tangent bundle $T M$. We can extend, at least over invariant control sets $C$ of $(2.1)$, the ${ }_{\mathbf{p}} D_{i}$ to ${ }_{T} D_{i} \subset T M$ by taking in each $T_{x} M$ the corresponding subspaces over $\mathbf{P}_{x} D_{i}:=\left.\mathbf{p} D_{i}\right|_{x} M$. For a control set $\mathbf{p} D$ in $\mathbf{P} M$, and its extension $T_{T} D$ in $T M$ define its lift to $\mathcal{U} \times \mathbf{P} M$ (and $\mathcal{U} \times T M$ respectively) to be

$$
\mathbf{P} \mathcal{D}=c \ell\{(u, \mathbf{P} x) ; \mathbf{P} \varphi(t, P x, u) \in \operatorname{int} \mathbf{p} D \text { for all } t \in \mathbf{R}\}
$$

and similarly for ${ }_{T} \mathcal{D}$. The question arises, whether the $T_{T} \mathcal{D}$ 's define an invariant subbundle decomposition of $(\mathcal{U} \times T M, T \phi)$. Unfortunately, this is in general not true, as the following simple example shows:

\subsection{EXAMPLE:}

Consider the following 2-dimensional system, linearized around a rest point (hence we give only the second component of (2.5))

$$
\dot{v}(t)=\left(\begin{array}{ll}
1 & 0 \\
0 & 0
\end{array}\right) v(t)+u_{1}(t)\left(\begin{array}{ll}
0 & 1 \\
1 & 0
\end{array}\right) v(t)+u_{2}(t)\left(\begin{array}{ll}
0 & 0 \\
0 & 1
\end{array}\right) v(t)
$$

with $U=\left[0, \frac{1}{2}\right] \times[1,2]$. To describe the control sets of the projected system on $P$, parametrize $\mathbf{P}$ via the angle as $\mathbf{P}=\left\{\eta ; \frac{-\pi}{2}<\eta \leq \frac{\pi}{2}\right\}$. Then the set $\mathbf{P}_{\mathbf{P}}=\left(-\frac{\pi}{4}, 0\right)$ is the open main control set, and ${ }_{\mathbf{p}} D_{2}=\left[\frac{\pi}{4}, \frac{\pi}{2}\right]$ is the closed main control set. These sets are connected by a continuum of control sets (with empty interior), which are rest points on $\mathbf{P}$ of the equation corresponding to the controls $u_{1}(t) \equiv 0, u_{2}(t) \equiv 1$, compare 
Example 5.8 in Colonius and Kliemann $\left(1990^{b}\right)$. Now note that for constant controls $u_{1}(t) \equiv \alpha, u_{2}(t) \equiv \beta$ the eigenvalues of the systems matrix $\left(\begin{array}{ll}1 & \alpha \\ \alpha & \beta\end{array}\right)$ are given by

$$
\lambda_{1,2}=\frac{1+\beta}{2} \pm \sqrt{\alpha^{2}-\beta+\frac{1}{4}(1+\beta)^{2}},
$$

and we have one dimensional (real) eigenspaces for all $(\alpha, \beta) \in U$, if $\alpha \neq 0$ and $\beta \neq 1$. For $\alpha=0, \beta=1$ the (generalized) eigenspace is $R^{2}$. Therefore the sets ${ }_{T} \mathcal{D}_{1}$ and ${ }_{T} \mathcal{D}_{2}$ do not define a subbundle decomposition of $(\mathcal{U} \times T M, T \phi)$, because subbundles are necessarily constant dimensional.

In order to obtain the finest subbundle decomposition of $(\mathcal{U} \times T M, T \phi)$, which respects the control structure of the system (2.6) on $P M$, we have to introduce generalizations of control sets, called chain control sets:

3.7. Definition: A set $E \subset M$ is called a chain control set of the system (2.1) if

(i) for every $x \in E$ there exists $u \in \mathcal{U}$ such that $\varphi(t, x, u) \in E$ for all $t \in \mathbf{R}$,

(ii) for all $x, y \in E$, all $\varepsilon>0$, and all $T>0$ there are $k \in \mathrm{N}, x_{0}, \ldots, x_{k} \in M$, $u_{0}, \ldots, u_{k-1} \in \mathcal{U}$ and $t_{0}, \ldots, t_{k-1} \geq T$ with $x_{0}=x, x_{k}=y$ and $d\left(\varphi\left(t_{i}, x_{i}, u_{i}\right), x_{i+1}\right)<\varepsilon$ for $i=1, \ldots, k-1$. Here $d$ denotes the Riemannian metric on $M$.

(iii) $E$ is maximal with respect to these properties.

\subsection{LEMMA.}

(i) Chain control sets are closed, connected, and pairwise disjoint.

(ii) For every control set $D \subset M$ there exists a unique chain control set $E$ with $D \subset E$.

(iii) Chain control sets are ordered by the relation

$$
\begin{aligned}
& E_{i} \prec E_{j} \text { if there exists }(u, x) \in \mathcal{U} \times M \text { with } \omega^{*}(u, x) \subset E_{i} \\
& \text { and } \omega(u, x) \subset E_{j},
\end{aligned}
$$

where $\omega^{*}(u, x)$ denotes the $\alpha$-limit set of the trajectory $\varphi(\cdot, x, u)$, and $\omega(u, x)$ its $\omega$-limit set.

For a proof compare Colonius and Kliemann $\left(1990^{a}\right)$.

3.9. REMARK: While each control set is contained in some chain control set, it is possible that one chain control set may contain several control sets (even with nonvoid interior), compare e.g. Example 3.6, where $E=\mathbf{P}^{1}$ is the chain control set. Also points that are in no control set, may be contained in a chain control set. Control sets are chain control sets, if they satisfy a certain isolation property, see the discussion in Colonius and Kliemann $\left(1990^{\circ}\right)$, Section 4.

Define the lift of a chain control set $E \subset M$ of $(2.1)$ to $\mathcal{U} \times M$ by

$$
\mathcal{E}=\{(u, x) \in \mathcal{U} \times M ; \varphi(t, x, u) \in E \text { for all } t \in \mathbf{R}\}
$$


Similarly for the projected linearized system (2.6) on $P M$ we can define its chain control sets, which we denote by $\mathbf{p} E \subset \mathrm{P} M . \mathbf{p} E$ can be lifted to $\mathcal{U} \times \mathrm{P} M$ as above, and we define the extensions to $\mathcal{U} \times T M$ by

$$
{ }_{T} \mathcal{E}=\{(u, T x) \in \mathcal{U} \times T M ;(u, T x) \notin Z \Longrightarrow \mathbf{P} \varphi(t, T x, u) \in \mathbf{p} E \text { for all } t \in \mathbf{R}\},
$$

where $Z$ is the zero section of the bundle $T M$. The following theorem gives the desired decomposition result:

3.10. Theorem. Assume that $M$ is compact, and that $M$ is the chain control set of (2.1).

(i) The control system (2.6) on $\mathrm{P} M$ has $\ell$ chain control sets $\mathbf{p} E_{1}, \ldots, \mathbf{p} E_{\ell}$ with $1 \leq \ell \leq d=\operatorname{dim} M$. The order defined in (3.6) is a linear order, and we enumerate the sets such that $\mathbf{p} E_{1} \prec_{\mathbf{p}} \cdots \prec_{\mathbf{p}} \mathbf{p} E_{\ell}$.

(ii) The lifts ${ }_{T} \mathcal{E}_{i}, i=1, \ldots, \ell$ are invariant subbundles of $\mathcal{U} \times T M$ with $\mathcal{U} \times T M=$ ${ }_{T} \mathcal{E}_{1} \oplus \cdots \oplus \mathcal{E}_{\ell}$.

(iii) $\left\{\mathbf{p} \mathcal{E}_{1}, \ldots, \mathbf{p} \mathcal{E}_{\ell}\right\}$ is the (unique) finest Morse decomposition of the flow $(\mathcal{U} \times$ $\mathbf{P} M, \mathbf{P} \phi)$. 4.9.

A proof of these results can be found in Colonius and Kliemann $\left(1990^{b}\right)$, Theorem

Combining Theorems 3.5 and 3.10 we obtain the following

3.11. Corollary. Assume that (PH) holds, and let $C$ be a compact, invariant control set of (2.1). Then we have for the projected system (2.6) on PintC: Every chain control set $\mathbf{p} E_{j}$ contains a main control set $\mathbf{p} D_{i}$. In particular, $1 \leq \ell \leq k \leq d$ and int $\mathbf{p} E_{j} \neq \phi$ for all $j=1, \ldots, \ell$.

The proof is an obvious generalization of Theorem 5.5 in Colonius and Kliemann $\left(1990^{b}\right)$. A more precise characterization of the $\mathbf{p} D_{i}$ and $\mathbf{p} E_{j}$ in terms of eigenspaces of matrices in the systems group of the linearized system will be given in Section 5.

Using the preparatory material in this section, we now turn to the discussion of the Lyapunov exponents of (2.1) and their characterization via ergodic theory.

\section{Ergodic Theory for Lyapunov Exponents.}

Consider again the nonlinear control system

$$
\dot{x}(t)=X_{0}(x(t))+\sum_{i=1}^{m} u_{i}(t) X_{i}(x(t)), \quad t \in \mathbf{R}, \quad x(0)=x_{0} \in M
$$

and the induced systems (2.4) on $T M$, and (2.6) on PM. Again in order to avoid degenerate situations, we will assume that the Lie algebra condition (PH) on PM holds. Recall from Section 2. that the linearized flow $T \phi$ on $\mathcal{U} \times T M$ is of the form $T \phi=$ $(\phi, D \varphi)$, where $D \varphi$ is a cocycle associated with $\phi$. 
The (forward) Lyapunov exponent of the control flow $\phi$ on $\mathcal{U} \times M$ at $(u, x) \in \mathcal{U} \times M$ in the direction of $v \in T_{x} M, v \neq 0$, is then defined by

$$
\lambda(u, x, v)=\limsup _{t \rightarrow \infty} \frac{1}{t} \log \|D \varphi(t, x, u) v\| .
$$

Similarly, backward Lyapunov exponents $\lambda^{-}(u, x, v)$ for $t \rightarrow-\infty$ are defined. Note that, in general, the following facts hold for Lyapunov exponents (even of linear systems): $\lambda(u, x, v) \neq \lambda^{-}(u, x, v)$, the lim sup is not a lim, and (exponential) stability of the system does not necessarily imply stability of small perturbations of the vectorfield, see e.g. Cesari (1971) or Hahn (1967) for examples in the context of ordinary differential equations.

In order that these three properties do hold, Lyapunov has introduced the concept of regularity. This concept is best expressed in Oseledec multiplicative ergodic theorem (1968), where stationarity of the underlying flow $\phi$ (i.e. the existence of a $\phi$-invariant probability measure $P$ ) is assumed, together with a certain integrability condition on the cocycle. In this case, there is a $\phi$-invariant set of full $P$-measure, such that all points in this set are Lyapunov regular, compare e.g. Ruelle (1979) for a presentation of this theory, which is one cornerstone in modern entropy theory (cp. Mañé (1987)), ergodic and stability theory of stochastic systems (cp. Carverhill (1985)), Arnold and Wihstutz (1986)), stochastic bifurcation theory (cp. Arnold and Boxler (1989)), and other areas, as also demonstrated by this proceedings volume. In the stochastic case, where the flow $\phi$ is induced by a nondegenerate diffusion process (over the Wiener space $(\Omega, \mathcal{F}, P)$ ) with invariant probability measure $\mu$ on $M$, it can actually be shown, for all $v$ fixed, that the Lyapunov exponents are $P \otimes \mu$-almost surely constant, i.e. with probability one only one Lyapunov exponent will be realized, compare Arnold et al. (1986) for the linear case, and San Martin and Arnold (1986) for the nonlinear situation.

For control systems, the situation is completely different, because one always has to deal with nonregular points: Let the vector fields $X_{0}, \ldots, X_{m}$ in (2.1) be linear, i.e. the system is of the form

$$
\dot{x}(t)=A_{0} x(t)+\sum_{i=1}^{m} u_{i}(t) A_{i} x(t)
$$

with non zero constant $d \times d$ matrices $A_{0} \ldots A_{m}$. Let the space of admissible control values $U \subset \mathrm{R}^{m}$ be the product of intervals $I_{i}$, i.e. $U=\mathrm{X}_{i=1}^{m} I_{i}$, such that at least one $I_{i}$ has nonvoid interior. Then there exists an admissible control function $u(t)=\left(u_{i}(t)\right) \in \mathcal{U}$, such that the matrix function $A_{0}+\sum_{i=1}^{m} u_{i}(t) A_{i}$ is not Lyapunov regular, compare e.g. Cesari (1971) or Hahn (1967). Since we are interested in the entire Lyapunov spectrum for all $(u, x, v) \in \mathcal{U} \times T M, v \neq 0$, we cannot use versions of Oseledeč's theorem. In the following, we will relate the Lyapunov spectrum of (2.1)

$$
\boldsymbol{\Sigma}=\{\lambda(u, x, v) ;(u, x, v) \in \mathcal{U} \times T M, v \neq 0\}
$$

to the control sets of the projected, linearized system (2.6) on PM via ergodic theory. More detailed information about the linear case (4.2) is presented in Section 5. 
Our starting point is the following formula for the Lyapunov exponents

$$
\lambda(u, x, v)=\limsup _{t \rightarrow \infty} \frac{1}{t} \int_{0}^{t} q\left(u(\tau), \mathbf{P} \varphi\left(\tau, \mathbf{P}_{x}, u\right)\right) d \tau,
$$

where again $\mathbf{P} \varphi(\cdot, \mathrm{P} x, u)$ denotes the solution of the projected system (2.6) on $\mathbf{P} M$, with initial value $\mathbf{P} \varphi(0, \mathbf{P} x, u)=(x, v) \in \mathbf{P} M$, i.e. $v \in \mathbf{P}_{x} M$. Note that $\lambda(u, x, \alpha v)=$ $\lambda(u, x, v)$ for all $\alpha \in \mathbf{R}, \alpha \neq 0$, and hence it suffices to consider (4.4) on $\mathcal{U} \times \mathbf{P} M$. The function $q$ is of the form

$$
q(u, x, v)=\left\langle v, \nabla X_{0}(x)\right\rangle+\sum_{i=1}^{m} u_{i}\left\langle v, \nabla X_{i}(x)\right\rangle,
$$

where $\langle\cdot, \cdot\rangle$ denotes the (Riemannian) inner product on $T M$, and $\nabla$ is the Riemannian covariant derivative (compare e.g. Baxendale (1986) for details).

From Formula (4.4) it is clear that the Lyapunov exponents are defined on the tail of the trajectory $(u(\cdot), \mathbf{P} \varphi(\cdot, \mathbf{P} x, u))$, therefore we first examine the $\omega$-limit sets of the flow $\mathbf{P} \phi$ on $\mathcal{U} \times \mathbf{P} M$. In the following, we will assume that the manifold $M$ is compact, but our results generalize immediately to the case, where $\cup\{\bar{D}, D$ is a control set of (2.1) $\}$ is a bounded set in the manifold metric on $M$, compare Colonius and Kliemann $\left(1990^{c}\right)$.

4.1. Definition: Let $(S, \Psi)$ be a continuous dynamical system on a compact metric space $S$. The $\omega$-limit set of $x \in S$ is defined as

$$
\omega(x):=\left\{y \in S ; \text { there exists } t_{k} \rightarrow \infty \text { in } \mathbf{R} \text { with } \Psi\left(t_{k}, x\right) \rightarrow y\right\} .
$$

For a control set $\mathbf{P} D$ of (2.6), not necessarily with nonvoid interior, we define its positive lift to $\mathcal{U} \times \mathrm{P} M$ as

$$
\mathbf{P} \mathcal{D}^{+}:=c \ell\{(u, \mathbf{P} x) \in \mathcal{U} \times \mathbf{P} M ; \mathbf{P} \varphi(t, \mathbf{P} x, u) \in \mathbf{P} D \text { for all } t \geq 0\} .
$$

Under our assumption that $M$ is compact, we obtain the following properties of the limit sets of $\mathbf{P} \phi$ :

\subsection{LeMMA.}

(i) For all $(u, \mathbf{P} x) \in \mathcal{U} \times \mathbf{P} M$ the limit sets $\omega(u, \mathbf{P} x)$ are connected, compact, and $\mathbf{P} \phi$-invariant, hence they contain minimal $\mathbf{P} \phi$-invariant sets.

(ii) For all $(u, \mathbf{P} x) \in \mathcal{U} \times \mathbf{P} M$ there exists a chain control set $\mathbf{p} E$ of (2.6) such that $\omega(u, \mathbf{P} x) \subset \mathbf{P} \mathcal{E}$, in particular $\pi_{\mathbf{P} M} \omega(u, \mathbf{P} x) \subset \mathbf{P} E$.

(iii) For all $(u, \mathbf{P} x) \in \mathcal{U} \times \mathbf{P} M$ there exists a control set $\mathbf{p} D$ of (2.6) such that $\omega(u, \mathbf{P} x) \cap$ $\mathbf{P}^{+} \neq \phi$, in particular $\pi_{\mathbf{P} M} \omega(u, \mathbf{P} x) \cap \mathbf{p} D \neq \phi$.

(iv) Let $\mathcal{W} \subset \mathcal{U} \times \mathbf{P} M$ be a minimal $\mathbf{P} \phi$-invariant set, then there exists a control set $\mathbf{p} D$ of (2.6) such that $\mathcal{W} \subset \mathbf{p} \mathcal{D}^{+}$.

(v) Let $\mathbf{p} D \subset \mathbf{P} M$ be a control set of (2.6), then for any $\mathbf{P} y \in \overline{\mathbf{p} D}$ there exists $(u, \mathbf{P} x) \in \mathcal{U} \times \mathbf{P} M$ such that $\mathbf{P} y \in \pi_{\mathbf{P} M} \omega(u, \mathbf{P} x)$.

(vi) The set $\left\{(u, x) \in \mathcal{U} \times M ; \pi_{M} \omega(u, x) \subset \operatorname{int} C\right.$ for some invariant control set $\left.C\right\}$ is open and dense in $\mathcal{U} \times M$. 
The proof of (i)-(v) can be found in Colonius and Kliemann (1990 ${ }^{\circ}$, Lemma 5.3), and (vi) is proved in Colonius and Kliemann $\left(1991^{a}\right)$.

Next we construct and characterize $\mathbf{P} \phi$-invariant probability measures via the KrylovBogolyubov construction: Let $C(\mathcal{U} \times \mathbf{P} M, \mathbf{R})$ denote the continuous functions from $\mathcal{U} \times \mathbf{P} M$ into R. For $(u, \mathbf{P} x) \in \mathcal{U} \times \mathbf{P} M$ consider the Cesaro limits for time sequences $t_{k} \rightarrow \infty$

$$
\lim _{t_{k} \rightarrow \infty} \frac{1}{t_{k}} \int_{0}^{t_{k}} F\left(\theta_{\tau} u, \mathbf{P} \varphi(\tau, \mathbf{P} x, u)\right) d \tau=\int_{u \times \mathbf{P} M} F(v, y) d \mu_{u, \mathbf{P} x}
$$

for all $F \in C(\mathcal{U} \times \mathrm{P} M, \mathbf{R})$. We will use the following notations for a continuous dynamical system $(S, \Psi)$ :

$\mathcal{M}_{\Psi}^{+}$: set of $\Psi$-invariant probability measures for $t \geq 0$,

$$
\Sigma_{\Psi}^{e}=\left\{s \in S \text {; the Krylov-Bogolyubov measure } \mu_{s}\right. \text { is independent of the }
$$
sequence $t_{k}$ and ergodic\},

$\Sigma_{\Psi}^{s}=\left\{s \in \Sigma_{\Psi}^{e} ; s \in \operatorname{supp} \mu_{s}\right\}$.

4.3. REMARK: The following properties of $\Psi$-invariant measures are well known for compact spaces $S$, compare e.g. Mañé (1987), Chapter II.6:

(i) $\Sigma_{\Psi}^{s} \neq \phi$, and $\Sigma_{\Psi}^{s}$ has total measure with respect to $\mathcal{M}_{\Psi}^{+}$, i.e. $\mu\left(\Sigma_{\Psi}^{s}\right)^{\mathrm{c}}=0$ for all $\mu \in \mathcal{M}_{\Psi}^{+}$. (Here $A^{c}$ denotes the complement of a set $A$.)

(ii) Each $\mu \in \mathcal{M}_{\Psi}^{+}$has an ergodic decomposition: Each $F \in L^{1}(S, \mu)$ is $\mu_{\text {s }}$-integrable for $\mu$-almost all $s \in \Sigma_{\Psi}^{s}$ and $\int\left(\int_{S} F d \mu_{s}\right) d \mu=\int_{S} F d \mu$.

In Crauel (1986) a characterization of (stochastic) Lyapunov exponents in terms of invariant measures was given for linear stochastic systems. We will now generalize these results to nonlinear control systems and, with the help of Lemma 4.2, provide additional insight into the support of invariant measures, and thus into the structure of realizable Lyapunov exponents. In order to use the Krylov- Bogolyubov measures of the control flow $\mathbf{P} \phi$, we first have to lift the function $q: U \times \mathbf{P} M \rightarrow \mathbf{R}$, defined in (4.5) to a function on $\mathcal{U} \times \mathbf{P} M:$ Define for $(u, \mathbf{P} x) \in \mathcal{U} \times \mathbf{P} M$

$$
Q(u, \mathbf{P} x)=\liminf _{h \downarrow 0} \frac{1}{h} \int_{0}^{h} q(u(\tau), \mathbf{P} \varphi(\tau, \mathbf{P} x, u)) d \tau .
$$

\subsection{LEMMA.}

(i) The function $Q: \mathcal{U} \times \mathbf{P} M \rightarrow \mathbf{R}$ is measurable and bounded.

(ii) For all $\left(u, P_{x}\right) \in \mathcal{U} \times \mathbf{P} M$

$$
\limsup _{t \rightarrow \infty} \frac{1}{t} \int_{0}^{t} q(u(\tau), \mathbf{P} \varphi(\tau, \mathbf{P} x, u)) d \tau=\limsup _{t \rightarrow \infty} \frac{1}{t} \int_{0}^{t} Q\left(\mathbf{P} \phi_{\tau}(u, \mathbf{P} x)\right) d \tau .
$$


Proof:

(i) Boundedness of $Q$ follows from the boundedness of $q$ on the compact space $U \times$ $P M$. Furthermore, $Q$ is the "lim inf" of continuous functions $(u, \mathbf{P} x) \mapsto \frac{1}{h} \int_{0}^{h} q(u(\tau), \mathbf{P} \varphi(\tau, \mathbf{P} x, u)) d \tau$ and therefore is measurable.

(ii) It suffices to show that for all $T>0$ we have $\int_{0}^{T} q d \tau=\int_{0}^{T} Q d \tau$ : By definition it holds that

$$
\int_{0}^{T} Q\left(\mathbf{P} \phi_{\tau}(u, \mathbf{P} x)\right) d \tau=\int_{0}^{T} \liminf _{h \downarrow 0} \frac{1}{h} \int_{0}^{h} q(u(\tau+\sigma), \mathbf{P} \varphi(\tau+\sigma, \mathbf{P} x, u)) d \sigma d \tau,
$$

and the function $t \mapsto q(u(t), \mathbf{P} \varphi(t, \mathbf{P} x, u))$ is Lebesgue-integrable on $[0, T]$. Therefore, for almost all $\tau \in[0, T]$ the $\operatorname{limit}_{h \downarrow 0} \lim _{h} \frac{1}{h} \int_{0}^{h} q(u(\tau+\sigma), \mathbf{P} \varphi(\tau+\sigma, \mathbf{P} x, u)) d \sigma$ exists and equals $q(u(\tau), \mathbf{P} \varphi(\tau, \mathbf{P} x, u))$, which proves the lemma.

4.5. Theorem. For each $(u, P x) \in \mathcal{U} \times \mathbf{P} M$ there exists an invariant probability measure $\mu_{u, \mathbf{P}_{x}}$ with supp $\mu_{u, \mathbf{P}_{x}} \subset \omega(u, \mathbf{P} x)$ such that

(i) $\lambda(u, x, v)=\int_{\omega(u, \mathbf{P} x)} Q(w, \mathbf{P} y) d \mu_{u, \mathbf{P} x}=\int_{\omega(u, \mathbf{P} x)} \lambda(w, \mathbf{P} y) d \mu_{u, \mathbf{P}_{x}}$,

(ii) there exists a chain control set $\mathbf{P} E$ of (2.6) such that for all ( $w, \mathbf{P} y) \in \operatorname{supp} \mu_{u, \mathbf{P} x}$ we have $\mathbf{P} \varphi(t, \mathbf{P} y, w) \in \mathbf{P} E \cap \pi_{\mathbf{P} M} \omega(u, \mathbf{P} x)$ for all $t \geq 0$,

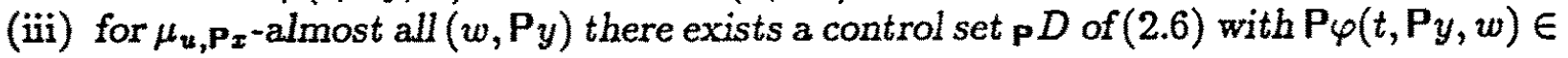
$\mathbf{P} D \cap \pi_{\mathbf{P} M} \omega(u, \mathbf{P} x)$ for all $t \geq 0$,

(iv) if $\mu_{u, \mathbf{P x}}$ is ergodic, then the control set $\mathbf{p} D$ in (iii) is unique, and $\lambda(w, \mathbf{P} y)$ is

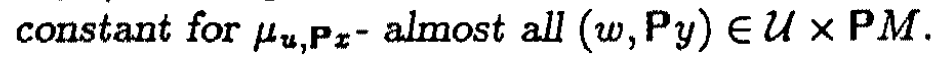

\section{PROOF:}

(i) This follows directly from Lemma 4.4 and Birkhoff's ergodic theorem.

(ii) is an immediate consequence of Lemma 4.2(ii).

(iii) follows from Lemma 4.2(iii) and the fact that for all $(u, \mathrm{P} x) \in \mathcal{U} \times \mathrm{P} M$ there exists a set $\Gamma \subset \operatorname{supp} \mu_{u, \mathbf{P}_{x}}$ with $\mu_{u}, \mathbf{P}_{x} \Gamma=1$, such that for all $(w, \mathbf{P} y) \in \Gamma$ there is a control set $\mathbf{P} D \subset \mathbf{P} M$ with $\mathbf{P}_{\varphi}(t, \mathbf{P} y, w) \subset \mathbf{P} D \cap \pi_{\mathbf{P} M} \omega(u, \mathbf{P} x)$ for all $t \geq 0$, compare Theorem 5.5(ii) in Colonius and Kliemann $\left(1990^{a}\right)$.

(iv) This is implied by Lemma 4.2(iv), and again Birkhoff's ergodic theorem.

The following result is now a direct consequence of Theorem 4.5 and Remark 4.3. In particular, it shows that all Lyapunov exponents can be obtained as integrals over Lyapunov exponents of regular elements. 


\subsection{Corollary.}

(i) For all $(u, \mathbf{P} x) \in \sum_{\mathbf{P} \phi}^{e}$ we have $\lambda(u, \mathbf{P} x)=\lim _{t \rightarrow \infty} \frac{1}{t} \int_{0}^{t} Q(\tau) d \tau=\lim _{t \rightarrow \infty} \frac{1}{t} \int_{0}^{t} q(\tau) d \tau=$ $\lambda(w, \mathbf{P} y)$ for $\mu_{u, \mathbf{P} x}$-almost all $(w, \mathbf{P} y)$.

(ii) For all $(u, \mathbf{P} x) \in \mathcal{U} \times \mathbf{P} M$ it holds that

$$
\lambda\left(u, \mathbf{P}_{x}\right)=\int_{\omega\left(u, \mathbf{P}_{x}\right)} \lambda\left(w, \mathbf{P}_{y}\right) d \mu_{u, \mathbf{P}_{\boldsymbol{x}},}
$$

where

$$
\lambda(w, \mathbf{P} y)=\lim _{t \rightarrow \infty} \frac{1}{t} \int_{0}^{t} q(\tau) d \tau
$$

Next we define the maximal and minimal Lyapunov exponent of the control system (2.1), which are realizable over a given set $\mathbf{P} A \subset \mathrm{P} M$ :

$$
\begin{aligned}
& \kappa(\mathbf{P} A)=\sup \{\lambda(u, \mathbf{P} x) ; \mathbf{P} \varphi(t, \mathbf{P} x, u) \in \mathbf{P} A \text { for all } t \geq 0\} \\
& \kappa^{*}(\mathbf{P} A)=\inf \{\lambda(u, \mathbf{P} x) ; \mathbf{P} \varphi(t, \mathbf{P} x, u) \in \mathbf{P} A \text { for all } t \geq 0\}
\end{aligned}
$$

4.7. Theorem. Let $\mathbf{p} \mathcal{A}^{+}=c \ell(u, \mathbf{P} x) \in \mathcal{U} \times \mathbf{P} M ; \mathbf{P} \varphi(t, \mathbf{P} x, u) \in \mathbf{p} A$ for all $\left.t \geq 0\right\}$ denote the positive lift of $\mathbf{p} A \subset \mathbf{P} M$ to $\mathcal{U} \times \mathbf{P} M$, and assume $\mathbf{p} \mathcal{A}^{+} \neq \phi$. Then there exist ergodic $\mathbf{P} \phi$-invariant measures $\mu_{\mathbf{P} A}$ and $\mu_{\mathbf{P} A}^{*}$ with supp $\mu_{\mathbf{P} A} \subset \mathbf{P} \mathcal{A}^{+}$and supp $\mu_{\mathbf{P} A}^{*} \subset$ $\mathbf{p} \mathcal{A}^{+}$such that

$$
\begin{gathered}
\kappa(\mathbf{P} A)=\int_{u \times \mathbf{P} M} Q(w, \mathbf{P} y) d \mu_{\mathbf{P} A}, \\
\kappa^{*}(\mathbf{P} A)=\int_{u \times \mathbf{P} M} Q\left(w, \mathbf{P}_{y}\right) d \mu_{\mathbf{P} A}^{*} .
\end{gathered}
$$

Furthermore, there are $(u, \mathbf{P} x) \in \mathbf{P} \mathcal{A}^{+},\left(u^{*}, \mathbf{P} x^{*}\right) \in \mathbf{P} \mathcal{A}^{+}$such that $\mu_{\mathbf{P} A}=\mu_{u, \mathbf{P}_{x}}$ and $\mu_{\mathbf{P} A}^{*}=\mu_{u^{*}, \mathbf{P}_{x^{*}}}$ where $\mu_{u, \mathbf{P}_{x}}$ and $\mu_{u^{*}, \mathbf{P}_{x^{*}}}$ denote again Krylov-Bogolyubov measures from $(u, \mathbf{P} x)$ and from $\left(u^{*}, \mathbf{P} x^{*}\right)$.

Proof: Note first of all that, because $\mathbf{p} \mathcal{A}^{+}$is closed and positively $\mathbf{P} \phi$-invariant, we have $\omega\left(u, \mathbf{P}_{x}\right) \subset \mathbf{P} \mathcal{A}^{+}$for all $\left(u, \mathbf{P}_{x}\right) \in \mathbf{p} \mathcal{A}^{+}$. Hence, by Corollary 4.6.

$$
\kappa(\mathbf{p} A)=\sup \left\{\int_{\mathbf{r} \mathcal{A}^{+}} Q d \mu_{u, \mathbf{P}_{x}} ;\left(u, \mathbf{P}_{x}\right) \in \Sigma_{\mathbf{P} \phi}^{s} \cap \mathbf{P} \mathcal{A}^{+}\right\} .
$$

Now the Krylov-Bogolyubov measures from points in $\Sigma_{\mathbf{P}_{\phi}}^{\mathbf{s}_{\phi}}$ are extremal points of the convex set $\mathcal{M}_{\mathbf{P}_{\phi}}^{+}$(compare Mañé (1987), Section II.2), hence the measures from points in $\Sigma_{\mathbf{P}_{\phi}}^{\mathbf{s}_{\mathbf{P}}} \cap \mathcal{A}^{+}$are extremal in $\left\{\mu \in \mathcal{M}_{\mathbf{P} \phi}^{+}\right.$; supp $\left.\mu \subset \mathbf{P} \mathcal{A}^{+}\right\}$, which is a closed, convex 
set, hence there exist $\mu_{\mathbf{P} A}$ and $\mu_{\mathbf{P} A}^{*}$ as required. This proves the formula for $\kappa(\mathbf{P} A)$, and similarly for $\kappa^{*}(\mathbf{p} A)$.

By Remark 4.3 we have $\int_{\mathcal{A}^{+}} Q d \mu_{\mathbf{P} A}=\int_{\mathbf{P}^{+}}\left(\int Q d \mu_{u, \mathbf{P}_{x}}\right) d \mu_{\mathbf{P} A}$ with $\left(u, \mathbf{P}_{x}\right) \in \Sigma_{\mathbf{P}_{\phi}}^{\mathbf{s}_{\mathbf{P}}}$ almost surely with respect to $\mu_{\mathbf{P} A}$. Because $\mu_{\mathbf{P} A}$ is ergodic, $\int Q d \mu_{u, \mathbf{P}_{x}}$ is $\mu_{\mathbf{P} A}$-almost everywhere constant, i.e. $\int Q d \mu_{\mathbf{P} A}=\int Q d \mu_{u, \mathbf{P}_{x}}$ for $\mu_{\mathbf{P} A^{-}}$-almost all $(u, \mathbf{P} x)$, which proves the other claim of the theorem.

4.8. REMARK: (On time reversal and backward Lyapunov exponents.)

Consider now the control system (2.1) on the entire time axis R. Its associated time reversed system reads with $x^{*}(t)=x(-t)$ :

$$
\dot{x}^{*}(t)=-X_{0}\left(x^{*}(t)\right)-\sum_{i=1}^{m} u_{i}(t) X_{i}\left(x^{*}(t)\right), t \in \mathbf{R}, x(0)=x_{0} \in M
$$

Define the backward Lyapunov exponents of (2.1) by

$$
\lambda^{-}(u, x, v)=\limsup _{t \rightarrow-\infty} \frac{1}{t} \log \|D \varphi(t, x, u) v\| .
$$

Note that

$$
\begin{aligned}
\lambda^{*}(u, \mathbf{P} x) & :=\liminf _{t \rightarrow \infty} \frac{1}{t} \int_{0}^{t} q^{*}\left(u(\tau), \mathbf{P} \varphi^{*}(\tau, \mathbf{P} x, u)\right) d \tau \\
& =\liminf _{t \rightarrow \infty} \frac{1}{t} \int_{0}^{t}-q\left(u(\tau), \mathbf{P} \varphi^{*}(\tau, \mathbf{P} x, u)\right) d \tau \\
& =-\limsup _{t \rightarrow-\infty} \frac{1}{t} \int_{0}^{t} q(u(\tau), \mathbf{P} \varphi(\tau, \mathbf{P} x, u)) d \tau
\end{aligned}
$$

where $q^{*}$ and $\mathbf{P} \varphi^{*}$ correspond to the time reversed system (4.11). In particular, if the $\lim$ inf in the definition of $\lambda^{*}(u, \mathbf{P} x)$ is a limit, then $-\lambda^{*}(u, \mathbf{P} x)$ is a backward Lyapunov exponent of (2.1). Now for the time reversed system (4.11) all results developed above hold with the obvious changes, and the assumption ( $P H)$ holds for (4.11) iff it holds for (2.1).

We obtain from an extension of Birkhoff's ergodic theorem (cp. Mañé (1987), Corollary II.1.4): If the Krylov-Bogolyubov measure $\mu_{u, \mathbf{P x}}$ is ergodic, then

$$
\lambda(w, \mathbf{P} y)=\lim _{t \rightarrow \infty} \frac{1}{t} \int_{0}^{t} Q(\tau) d \tau=\lim _{t \rightarrow \infty} \frac{1}{t} \int_{0}^{t} Q(-\tau) d \tau=\lambda^{-}\left(w, P_{y}\right)
$$

for $\mu_{u, \mathbf{P} x}$-almost all $(w, \mathbf{P} y)$. Therefore, if $(u, \mathbf{P} x) \in \operatorname{supp} \mu_{u, \mathbf{P} x}$, then $\lambda(u, \mathbf{P} x)=$ $\lambda^{-}(u, \mathbf{P} x)$, which extends the results of Corollary 4.6 and Theorem 4.7. Note, however, that if $\lambda(u, x, v)=\lambda^{-}(u, x, v)$ for some $v \in T_{x} M$, then a basis $\left\{v_{1}, \ldots, v_{d}\right\}$ in 
$T_{x} M$ need not exist with $\lambda\left(u, x, v_{i}\right)=\lambda^{-}\left(u, x, v_{i}\right)$ for all $i=1, \ldots, d$, i.e. the point $(u, x) \in \mathcal{U} \times M$ need not be Lyapunov regular for the flow $\phi$.

4.9. REMARK: The connection of our results obtained so far with the multiplicative ergodic theorem of Oseledec is as follows: Consider the point $(u, \mathbf{P} x) \in \mathcal{U} \times \mathbf{P} M$ and the Krylov-Bogolyubov measure $\mu_{u, P_{x}}$. The projection of $\mu_{u, P_{x}}$ onto $\mathcal{U} \times M$ is a $\phi$ invariant measure, denoted by $\mu_{u, x}$. According to Oseledeč's theorem, there exists a set $\Gamma \subset \operatorname{supp} \mu_{u, x}$ with $\mu_{u, x} \Gamma=1$, such that all points in $\Gamma$ are Lyapunov regular. (Note that the set $\Gamma$ may be very thin from a topological point of view, compare e.g. Mañé (1987), p. 264.) Theorem 4.5 and Corollary 4.6 describe the way, in which the Lyapunov exponents $\lambda(u, x, v)$ can be obtained from the finite Lyapunov spectrum of regular elements of the flow $\left(\mathcal{U} \times M, \phi, \mu_{u, x}\right)$. In particular, if $\mu_{u, \mathbf{P}_{x}}$ is ergodic, then $\mu_{u, x}$ is ergodic, and hence the set $\{\lambda(w, y, v) ;(w, y) \in \Gamma\}$ consists of at most $d$ numbers, which are also the Lyapunov spectrum of $(u, x)$.

If the measure $\mu_{u, x}$ is not ergodic, then the (finitely many) numbers $\{\lambda(u, x, v) ; v \neq$ $0\}$ are $\mu_{u}, \mathbf{P}_{\boldsymbol{x}}$-averages over the Lyapunov spectrum of $\Gamma$, which now may depend on $(w, y) \in \Gamma$.

We are now ready to apply our results to the analysis of the Lyapunov spectrum

$$
\boldsymbol{\Sigma}=\{\lambda(u, x, v) ;(u, x, v) \in \mathcal{U} \times T M, v \neq 0\}
$$

of the control system (2.1). In control theory, one considers the dynamics, i.e. the vector fields $X_{0}, \ldots, X_{m}$, and the set of control values, i.e. $U \subset \mathbf{R}^{m}$, as given. The problem then is to find an admissible control function $u \in \mathcal{U}$, such that a certain goal (like controllability, stabilization, etc.) is achieved from an initial point $x \in M$. The goal in our context is to describe the Lyapunov exponents that can be realized from a point $x \in M$ using all $u \in \mathcal{U}$. The standing assumption is still that $(\mathrm{P} H)$ holds, and that $M$ is compact. We start analyzing the Lyapunov spectrum of (2.1) by considering it from the point of view of chain control sets.

Let $E \subset M$ be a chain control set of (2.1) and denote

$$
\mathcal{P} E=\left\{\mathrm{P} E ; \mathrm{P} E \text { is a chain control set of }(2.6) \text { and } \pi_{M \mathrm{P}} E \subset E\right\}
$$

Define the corresponding spectrum over $E$ as

$$
\boldsymbol{\Sigma}(E)=\left\{\lambda(u, x, v) ;(u, x) \in \mathcal{E} \subset \mathcal{U} \times M, v \in T_{x} M\right\}
$$

For a chain control set $\mathrm{P} E \subset P M$ of (2.6) denote its spectral interval by

$$
I(\mathbf{p} E)=\left[\kappa^{*}(\mathbf{p} E), \kappa(\mathbf{p} E)\right]
$$

where $\kappa$ and $\kappa^{*}$ are defined in (4.10). We have the following result: 


\subsection{THEOREM.}

(i) $\boldsymbol{\Sigma}(E) \subset \cup\{I(\mathbf{p} E) ; \mathbf{p} E \in \mathcal{P} E\}=: I(E)$.

(ii) $\Sigma \subset \cup\{I(E) ; E$ is a chain control set of (2.1)\}.

(iii) For a chain control set $E \subset M$ of (2.1), let “ $\prec_{\mathbf{p}}$ " be the order in $\mathcal{P} E$ as defined in (3.6). Then for two sets $\mathbf{p} E_{1}, \mathbf{p} E_{2}$ in $\mathcal{P} E$ we have: $\mathbf{p} E_{1} \prec_{\mathbf{p}} \mathbf{p} E_{2}$ implies $I\left(\mathbf{p} E_{1}\right) \leq I\left(\mathbf{p} E_{2}\right)$, in the sense that $\kappa^{*}\left(\mathbf{p} E_{1}\right) \leq \kappa^{*}\left(\mathbf{p} E_{2}\right)$ and $\kappa\left(\mathbf{p} E_{1}\right) \leq \kappa\left(\mathbf{p} E_{2}\right)$.

(iv) In particular, if $M$ is the chain control set of (2.1), then $\boldsymbol{\Sigma} \subset \bigcup_{j=1}^{\ell} I\left(\mathbf{p} E_{j}\right)$, where the $\mathbf{p} E_{j}$ are as in Theorem 3.10.

\section{PROOF:}

(i) Denote by $\pi: \mathrm{P} M \rightarrow M$ the projective bundle over $M$, and note that $(u, x) \in \mathcal{E}$ implies $\mathbf{P} \varphi(t,(x, v), u) \in \pi^{-1}[E]$ for all $t \in \mathbf{R}$, all $v \in \mathbf{P}_{\boldsymbol{x}} M$. By Lemma 4.2(ii) we therefore have that $\omega(u, x, v) \subset \mathbf{p} \mathcal{E}$ for some chain control set $\mathbf{p} E$ in $\mathcal{P} E$. Theorem 4.5(i) now says that $\lambda(u, x, v) \in I(\mathbf{p} E)$.

(ii) For $(u, x) \in \mathcal{U} \times M$ the $\omega$-limit set $\omega(u, x)$ is contained in some lifted chain control set $\mathcal{E}$ and $\pi_{M} \omega(u, x) \subset E$ by Lemma $4.2($ ii). Hence the proof goes through as in (i).

(ii), (iv) This follows from Theorem 3.10 and its proof in Colonius and Kliemann $\left(1990^{b}\right)$.

The spectral intervals $I(\mathbf{p} E)$ over different chain control sets need not be disjoint, as the following simple example shows:

\subsection{EXAMPLE:}

Consider the following 2-dimensional linearized system

$$
\dot{v}(t)=u_{1}(t)\left(\begin{array}{ll}
1 & 0 \\
0 & 1
\end{array}\right) v(t)+u_{2}(t)\left(\begin{array}{ll}
0 & 1 \\
0 & 0
\end{array}\right) v(t)+u_{3}(t)\left(\begin{array}{ll}
0 & 0 \\
1 & 0
\end{array}\right) v(t)
$$

with $U=[0,2] \times\left[\frac{1}{2}, 1\right] \times\left[\frac{1}{2}, 1\right]$. The control sets of the projected system on the projective space $\mathbf{P}$ in $\mathbf{R}^{2}$ are given by

$$
\begin{aligned}
& \mathbf{P} D_{1}=\pi_{\mathbf{P}}\left\{\left(\begin{array}{l}
v_{1} \\
v_{2}
\end{array}\right) \in \mathbf{R}^{2} ; v_{2}=\alpha v_{1}, \alpha \in\left(-\sqrt{2},-\sqrt{\frac{1}{2}}\right)\right\}, \\
& \mathbf{P} D_{2}=\pi_{\mathbf{P}}\left\{\left(\begin{array}{l}
v_{1} \\
v_{2}
\end{array}\right) \in \mathbf{R}^{2} ; v_{2}=\alpha v_{1}, \alpha \in\left[\sqrt{\frac{1}{2}}, \sqrt{2}\right]\right\},
\end{aligned}
$$

where $\pi_{\mathbf{P}}$ denotes the natural identification of points in $\mathbf{R}^{2}$ as subspaces, i.e. as elements in $P$. The chain control sets are

$$
\mathbf{p} E_{1}=\mathbf{p} \bar{D}_{1}, \quad \mathbf{p} E_{2}=\mathbf{p} D_{2}
$$

compare Colonius and Kliemann (1990c, Theorem 3.16) for a general technique to compute control sets and chain control sets for systems with 1-dimensional state space, and Colonius and Kliemann $\left(1991^{b}\right)$ for the details on this example. 
For constant $u \in U$ denote by $A(u)=\left(\begin{array}{ll}u_{1} & u_{2} \\ u_{3} & u_{1}\end{array}\right)$ the possible right hand sides of (4.16). Let $\lambda_{1}(u)$ and $\lambda_{2}(u)$ be the (real parts of the) eigenvalues of $A(u)$ with $\lambda_{1}(u) \leq$ $\lambda_{2}(u)$. Then $\left\{\lambda_{1}(u), u \in U\right\}=\left[-1, \frac{3}{2}\right]$ and $\left\{\lambda_{2}(u) ; u \in U\right\}=\left[\frac{1}{2}, 3\right]$. Since these sets are contained in the spectral intervals over $\mathbf{p} E_{1}$ and $\mathbf{p} E_{2}$, we obtain $I\left(\mathbf{p} E_{1}\right) \supset\left[-1, \frac{3}{2}\right]$ and $I\left(\mathbf{p} E_{2}\right) \supset\left[\frac{1}{2}, 3\right]$, in particular $I\left(\mathbf{p} E_{1}\right)$ and $I\left(\mathbf{p} E_{2}\right)$ overlap in an entire interval.

Theorem 4.10 says that, in order to find the entire Lyapunov spectrum $\boldsymbol{\Sigma}$ of a nonlinear control system (2.1), one only has to consider the spectrum over the chain control sets $E$ of (2.1). In particular, take any $x \in M$ and any chain control set $E \subset M$ with $\overline{\mathcal{O}+(x)} \cap E=\phi$. Then $\left\{\lambda(u, x, v) ; u \in \mathcal{U}, v \in T_{x} M\right\} \cap I(E) \neq \phi$, i.e. some Lyapunov exponent in $I(E)$ can be realized from $x \in M$, if $E$ can approximately be reached from $x$. A similar statement holds for $\{\lambda(u, x, v) ; u \in \mathcal{U}\}$ with given $(x, v) \in \mathbf{P}_{x} M$, with respect to reachability in the projected system (2.6). This, of course, does not mean that all Lyapunov exponents in $I(E)$ can be realized from $x \in M$, if $\overline{\mathcal{O}^{+}(x)} \cap E \neq \phi$, compare e.g. Colonius and Kliemann $\left(1991^{b}\right)$ for a counter example. However, the control structure of (2.1) and (2.6) gives more information on the Lyapunov exponents that can be realized from $x \in M$ or $\mathrm{P}_{x} \in \mathrm{P}_{x} M$. This is the topic we will discuss throughout the rest of this section.

Let $D \subset M$ be a control set of (2.1) with int $D \neq \phi$, and denote

$$
\begin{gathered}
\mathcal{P} D=\{\mathbf{p} D ; \mathbf{p} D \text { is a control set of }(2.6) \text { with } \\
\text { int } \left.\mathbf{p} D \neq \phi \text { and } \pi_{M} \mathbf{p} D \subset D\right\} .
\end{gathered}
$$

Define the spectrum over $\mathbf{p} D$ and $D$ by

$$
\begin{aligned}
& \boldsymbol{\Sigma}(\mathbf{P} D)=\left\{\lambda(u, x, v) ;(x, v) \in \mathbf{P} D \text { and } \pi_{\mathbf{P} M} \omega(u, x, v) \subset \operatorname{int} D\right\} \\
& \boldsymbol{\Sigma}(D)=\cup\{\boldsymbol{\Sigma}(\mathbf{P} D) ; \mathbf{p} D \in \mathcal{P} D\}
\end{aligned}
$$

\subsection{THEOREM.}

(i) The closure of the $\boldsymbol{\Sigma}(\mathbf{p} D)$ are intervals. Furthermore, for all $(x, v) \in \mathbf{p} D$ we have $\{\lambda(u, x, v) ; u \in \mathcal{U}\} \supset \boldsymbol{\Sigma}(\mathbf{p} D)$, i.e. the Lyapunov exponents in $\boldsymbol{\Sigma}(\mathbf{p} D)$ can be realized from all $(x, v) \in \mathbf{p} D$.

(ii) For all $(x, v) \in \mathcal{O}^{-}(\mathbf{p} D)$ we have $\{\lambda(u, x, v) ; u \in \mathcal{U}\} \supset \boldsymbol{\Sigma}(\mathbf{p} D)$, where $\mathcal{O}^{-}(\mathbf{p} D)$ denotes the (open) negative orbit of the set $p D$ for the control system (2.6).

(iii) Let " $\prec_{\mathbf{P}}$ " be the order on the control sets of (2.6). If for some $(x, v) \in \mathbf{P} M$ we have $\mathcal{O}^{+}(x, v) \cap \mathbf{p} D$ then $\{\lambda(u, x, v) ; u \in \mathcal{U}\} \supset \cup\left\{\boldsymbol{\Sigma}\left(\mathbf{p} D^{\prime}\right) ; \operatorname{int} \mathbf{p} D^{\prime} \neq \phi\right.$ and $\left.\mathbf{p} D \prec \mathbf{p} \mathbf{p} D^{\prime}\right\}$. In particular for each $(x, v) \in \mathbf{P} M$ there exists an invariant control set ${ }_{\mathbf{P}} C \subset \mathbf{P} M$ such that $\{\lambda(u, x, v) ; u \in \mathcal{U}\} \supset \boldsymbol{\Sigma}(\mathbf{P} C)$.

(iv) For $x \in M$ let $D \subset M$ be a control set with $\mathcal{O}^{+}(x) \supset D$, and int $D \neq \phi$. Then $\left\{\lambda(u, x, v) ; u \in \mathcal{U}, v \in P_{x} M\right\} \supset \Sigma(D)$, and similarly for the results in (iii).

(v) The set $\{(u, x, v) \in \mathcal{U} \times T M ; \lambda(u, x, v) \in \boldsymbol{\Sigma}(\mathbf{p} C)$ for some invariant control set ${ }_{\mathrm{P}} C$ in $\left.\mathrm{P} M\right\}$ contains an open and dense subset of $\mathcal{U} \times T M$. 
ProOF:

(i) For the first claim see Colonius and Kliemann $\left(1991^{b}\right)$. Let $\lambda=\lambda\left(u^{\prime}, x^{\prime}, v^{\prime}\right) \in$ $\boldsymbol{\Sigma}(\mathbf{p} D)$, i.e. $\left(x^{\prime}, v^{\prime}\right) \in$ int $\mathbf{p} D$. Then, by Lemma 3.3(i), for each $(x, v) \in_{\mathbf{p}} D$ there exists a control $w \in \mathcal{U}$ such that $\mathbf{P} \varphi\left(t^{\prime},(x, v), w\right)=\left(x^{\prime}, v^{\prime}\right)$. Define the composite control $w^{\prime}$ as

$$
w^{\prime}(t)= \begin{cases}w(t) & \text { for } t \in\left[0, t^{\prime}\right] \\ u^{\prime}\left(t-t^{\prime}\right) & \text { for } t \in\left(t^{\prime}, \infty\right) .\end{cases}
$$

Then $\lambda\left(w^{\prime}, x, v\right)=\lambda\left(u^{\prime}, x^{\prime}, v^{\prime}\right)$.

(ii) This is proved in the same manner as (i).

(iii) The first statement follows from the definition of the order $\prec_{\mathbf{p}}$, compare Definition (3.2). The statement about invariant control sets follows from the discussion after (3.2) and the fact that under (PH) invariant control sets of (2.6) always have nonvoid interior, compare Lemma 3.3.

(iv) follows directly from (i)-(iii).

(v) By Lemma 4.2(vi) and Theorem 4.5(v) the set $\{(u, x, v) \in \mathcal{U} \times T M$;

$\pi_{\mathbf{P} M} \omega(u, x, v) \subset \operatorname{int}_{\mathbf{P}} C, \mathbf{P} C$ some invariant control set in $\left.\mathbf{P} M\right\}$ is open and dense. For all these $(u, x, v)$ one has $\lambda(u, x, v) \in \Sigma\left({ }_{\mathbf{p}} C\right)$.

4.13. Corollary. Let $C$ be a (compact) invariant control set of (2.1). Then for each $(x, v) \in \operatorname{Pint} C$, the projective bundle over int $C$, there exists a unique index $i_{0}$ such that $i_{0}=\min \left\{i \in\{1 \ldots k\} ; \mathcal{O}^{+}(x, v) \cap \mathbf{p} D_{i} \neq \phi\right\}$, where the $\mathbf{p} D_{i}$ are defined in Theorem 3.5. Furthermore, $\{\lambda(u, x, v) ; u \in \mathcal{U}\} \supset \Sigma\left(\mathbf{p} D_{i}\right)$ for all $i \geq i_{0}$, and $\Sigma\left(\mathbf{p} D_{i}\right) \leq \Sigma\left(\mathbf{p} D_{j}\right)$ if $i \leq j$, with the order defined as in Theorem 4.10.

The proof follows directly from Theorem 3.5 and Theorem 4.12(iii).

Because all control sets are contained in some (unique) chain control set (compare Lemma 3.8), each set $\boldsymbol{\Sigma}(\mathbf{p} D)$ (and $\boldsymbol{\Sigma}(D)$ ) is contained in some $I(\mathbf{p} E)$ (and $\boldsymbol{\Sigma}(E)$, respectively). In particular, over an invariant control set $C$ of (2.1), we have by Corollary 3.11 that each $I\left(\mathbf{p} E_{j}\right), j=1, \ldots \ell$ contains some $\Sigma\left(\mathbf{p} D_{i}\right)$ for $i=1 \ldots k$.

As the result of our discussion we see that the entire Lyapunov spectrum of a nonlinear control system (2.1) under Assumption (PH) can be found in spectral intervals over the chain control sets of (2.6). By Theorem 4.7, the boundary points of these intervals are themselves Lyapunov exponents, corresponding to some ergodic Krylov-Bogolyubov measures of $(\mathcal{U} \times \mathbf{P} M, \mathbf{P} \phi)$. On the other hand, the set of $(u, x, v) \in \mathcal{U} \times T M$ such that $\lambda(u, x, v)$ is attained over some invariant control set $\mathrm{P} C$ in $\mathrm{P} M$ contains an open and dense subset of $\mathcal{U} \times T M$.

Linearizations of nonlinear control systems around rest points lead to bilinear control systems, as described in Section 2. For these systems, the picture is much more complete, compare Colonius and Kliemann $\left(1990^{d}, 1991^{b}\right)$. In the next section, we will apply some of our results to the study of typical problems in the systems theory of bilinear systems (and linear feedback systems). A nonlinear example is considered in Section 6. 


\section{Some Applications to Linear and Bilinear Systems.}

Bilinear systems appear as linearization (with respect to the state variable) around a rest point of the nonlinear control system (2.1). Also, as we will see below, they are the feedback models of linear control systems. Lyapunov exponents of bilinear systems indicate the stability, stabilizability, and robustness properties of linear systems.

Consider the bilinear control system with bounded controls

$$
\dot{x}(t)=A_{0} x(t)+\sum_{i=1}^{m} u_{i}(t) A_{i} x(t), \quad t \in \mathbf{R}, \quad x(0)=x_{0} \in \mathbf{R}^{d}
$$

where $\left(u_{i}\right)=u \in \mathcal{U}$ as defined in Section 2. The $A_{0}, \ldots, A_{i}$ are given constant $d \times d$ matrices. (In Colonius and Kliemann $\left(1990^{d}\right)$, a more general system with bounded and unbounded controls is treated.)

In linear systems theory for equations of the type

$$
\dot{x}(t)=A x(t)+B u(t), \quad y(t)=C x(t), \quad u(t) \in U \subset \mathbf{R}^{m}
$$

(with $A d \times d, B d \times m, C k \times d$ constant matrices), one is usually interested in a feedback control $u(t)=F(t) C x(t)$ to achieve a certain goal, like stabilization. The systems equation then reads

$$
\dot{x}(t)=A x(t)+B F(t) C x(t)
$$

which is a system of the form (5.1). If the feedback matrix $F(t)$ can take any value in the $m \times k$-dimensional space, methods from linear algebra can be used to solve the typical control problems, see e.g. Wonham (1979). If, however, the set of admissible feedback values is bounded, nonlinear techniques, like e.g. the ones developed here, are appropriate.

As in Section 2. we consider the projected system on the projective space $\mathbf{P}^{d-1}$ in $\mathbf{R}^{d}$, and equation (2.7) reads now

$$
\dot{s}(t)=h(u(t), s(t))=h_{0}(s)+\sum_{i=1}^{m} u_{i}(t) h_{i}(s)
$$

with $h_{j}(s)=\left(A_{j}-s^{T} A_{j} s \cdot \mathrm{Id}\right) s, \quad j=0, \ldots, m$.

The Lyapunov exponents of $(5.1)$ are for $(u, x) \in \mathcal{U} \times \mathbf{R}^{d}, x \neq 0$

$$
\begin{aligned}
\lambda(u, x) & =\limsup _{t \rightarrow \infty} \frac{1}{t} \log |\varphi(t, x, u)| \\
& =\limsup _{t \rightarrow \infty} \frac{1}{t} \int_{0}^{t} q(u(\tau), s(\tau, s, u)) d \tau, \quad s=\frac{x}{\|x\|_{2}} \in \mathbf{P}^{d-1},
\end{aligned}
$$

where $|\cdot|$ is any norm in $\mathrm{R}^{d}$, and $q(u, s)=q_{0}(s)+\sum_{i=1}^{m} u_{i}(t) q_{i}(s)$, with $q_{j}(s)=s^{T} A_{j} s$ for $j=0, \ldots, m$. 
NotAtion: In this section we will use the variable $x$ for the state of the bilinear system (this was denoted by $v$ in Section 2.), $\varphi(\cdot, \cdot, \cdot)$ for the solution of $(5.1)$ (which corresponds to $T \varphi$ in Section 2.), $s$ and $s(\cdot, \cdot, \cdot)$ for the state on the projective space, and for the solution of (5.2) respectively (which correspond to $v \in \mathbf{P}_{x} M$ and $\mathbf{P} \varphi$ in Section 2.). Furthermore, the projective space $\mathbf{p} d-1$ in $\mathbf{R}^{d}$ will be denoted by $\mathbf{P}$. These changes are made so that we can use the usual notation in the literature for linear and bilinear systems. Similarly, for control sets (and chain control sets) on $\mathbf{P}$ we will simply use $D$ instead of $\mathbf{p}_{\mathbf{p}} D$ (and $E$ instead of $\mathbf{p} E$, respectively).

First of all, we will characterize the control sets and chain control sets of (5.2) more precisely via generalized eigenspaces of elements in the systems semigroup. Denote again by $\mathcal{S}^{+}$the positive semigroup of (5.1) (compare (3.1)), and note that $g \in \mathcal{S}^{+}$acts on $\mathbf{P}$ in a natural way via $s \mapsto \frac{1}{|g s|} g s$. For $g \in \mathcal{S}^{+}$denote by $u(g) \in \mathcal{U}$ a (not necessarily unique) periodic control function, associated with $g$. For a main control set $D$ of (5.2) (i.e. a control set with nonvoid interior, compare Theorem 3.5) define

$$
D(u(g))=\{x \in \mathbf{P} ; s(t, x, u(g)) \in \bar{D} \text { for all } t \in \mathbf{R}\}
$$

Then we have the following supplement to Theorems 3.5 and 3.10 :

\subsection{Theorem. Assume that Assumption (H) holds for the system (5.2).}

(i) For every $g \in \operatorname{int}_{\mathcal{G}} \mathcal{S}$ and every $\lambda \in \operatorname{spec} g$ there exists a main control set $D \subset P$ such that the (generalized) eigenspace $E(g, \lambda)$ satisfies $\mathbf{P}(E(g, \lambda)) \subset$ int $D$; where for a subspace $X \subset \mathbf{R}^{d}$ its projection onto $\mathrm{P}$ is denoted by $\mathrm{P}(X)$. Furthermore, the interior of the main control sets consists exactly of those elements $x \in P$, which are eigenvectors for a (real) eigenvalue of some $g \in$ int $\mathcal{S}^{+}$.

(ii) For every $g \in \mathcal{S}^{+}$and every $\lambda \in$ spec $g$ there exists a main control set $D$ with $\mathbf{P}(E(g, \lambda)) \cap \bar{D} \neq \phi$, and for every main control set $D$ and every $g \in \mathcal{S}^{+}$there is a $\lambda \in$ spec $g$ with $\mathbf{P}(E(g, \lambda)) \cap \bar{D} \neq \phi$.

(iii) For every $g \in$ int $\mathcal{S}^{+}$and every main control set we have $D(u(g))=\mathbf{P}\left(\oplus_{\lambda} E(g, \lambda)\right)$, where the sum is taken over all $\lambda \in \operatorname{spec} g$ with $\mathbf{P}(E(g, \lambda)) \subset$ int $D$.

(iv) Let $E$ be a chain control set of (5.2) and denote by $\mathcal{E}$ its lift to $\mathcal{U} \times P$. Then $\mathcal{E}=c \ell\left\{(u(g), x) \in \mathcal{U} \times \mathbf{P} ; g \in\right.$ int $\mathcal{S}^{+}$and $x \in \bigoplus_{i} D_{i}(u(g))$ for $i$ with $\left.D_{i} \subset E\right\}$.

The proof of this theorem can be found in Colonius and Kliemann $\left(1990^{b}\right)$, Theorem 3.10, Theorem 3.13, and Theorem 5.6. (Note that under Assumption (H) int $_{\mathcal{C}} \mathcal{S} \neq \phi$. ) This theorem allows us to reduce the analysis of (main) control sets and chain control sets to eigenspaces of elements in the systems semigroup, and it also implies that the Lyapunov spectrum over the main control sets can be approximated by (real parts of) the eigenvalues of the $g \in$ int $\mathcal{S}^{+}$, i.e. by the Lyapunov exponents corresponding to piecewise constant, periodic control functions. But the right hand side of (5.1) for this class of controls is Lyapunov regular. We continue by stating some implications of these facts for the stabilization of (5.1). We will be concerned here only with the extremal Lyapunov exponents of (5.1), the complete picture is described in Colonius and Kliemann $\left(1991^{b}\right)$, where also the existence of stabilizing feedback controls for bilinear systems is considered. 
Denote, as in (4.18), by $\Sigma(D)$ the Lyapunov spectrum over a main control set $D$ of (5.2). By Theorem 3.5 the main control sets of (5.2) are linearly ordered $D_{1} \prec \cdots \prec D_{k}$, and by Lemma 3.3 we have: $D_{1}=: C^{-}$is the open control set, $D_{k}=: C$ is the closed, invariant control set (with int $C \neq \phi$ ). It can actually be shown (compare Colonius and Kliemann $\left(1991^{b}\right)$ that the spectral sets $\Sigma(C)$ and $\Sigma\left(C^{-}\right)$are intervals, and by Theorem 4.7, their boundary points are Lyapunov exponents corresponding to ergodic, invariant measures of the flow $\left(\theta_{t}, s(t, \cdot, u)\right)$ on $\mathcal{U} \in \mathbf{P}$. Define the following extremal Lyapunov exponents

$$
\kappa=\sup \boldsymbol{\Sigma}(C), \quad \tilde{\kappa}=\inf \boldsymbol{\Sigma}(C), \quad \kappa^{*}=\inf \boldsymbol{\Sigma}\left(C^{-}\right) .
$$

As the following result shows, these three exponents can be characterized as global quantities of the control system (5.1), while sup $\Sigma\left(C^{-}\right)$has to be described through an invariant set of the flow on $\mathcal{U} \times \mathrm{P}$, compare again Colonius and Kliemann $\left(1991^{b}\right)$.

5.2. Theorem. Assume Assumption (H) for the system (5.2).

(i) $\kappa=\sup _{x \in \operatorname{int} C} \sup _{u \in \mathcal{U}} \lambda(u, x)=\sup _{x \neq 0} \sup _{u \in \mathcal{U}} \lambda(u, x)=\sup _{u \in \mathcal{U}} \lambda(u, x)$ for all $x \neq 0$,

(ii) $\tilde{\kappa}=\sup _{x \in \operatorname{int} C} \inf _{u \in \mathcal{U}} \lambda(u, x)=\sup _{x \neq 0} \inf _{u \in \mathcal{U}} \lambda(u, x)$,

(iii) $\kappa^{*}=\inf _{x \in C^{-}} \inf _{u \in \mathcal{U}} \lambda(u, x)=\inf _{x \neq 0} \inf _{u \in \mathcal{U}} \lambda(u, x)$.

(iv) For each $\varepsilon>0$ and all $x \in \mathrm{P}, \tilde{x} \in \operatorname{int} C, x^{*} \in C^{-}$there exist $g, \tilde{g}, g^{*} \in \operatorname{int} \mathcal{S}^{+}$ such that

$$
\lambda(u(g), x)>\kappa-\varepsilon, \quad \lambda(u(\tilde{g}), \tilde{x})<\tilde{\kappa}+\varepsilon, \quad \lambda\left(u\left(g^{*}\right), x^{*}\right)<\kappa^{*}+\varepsilon .
$$

The proof of this theorem is contained in Sections 4. and 5. of Colonius and Kliemann $\left(1990^{d}\right)$.

\subsection{REMARK:}

(i) It follows from Theorem 5.2 that $\kappa^{*} \leq \tilde{\kappa} \leq \kappa$. But the intervals $\Sigma(C)$ and $\Sigma\left(C^{-}\right)$ can overlap, i.e. $\tilde{\kappa}=\inf \Sigma(C)<\sup \Sigma\left(C^{-}\right)$is possible, compare Example 4.11.

(ii) The growth rates $\kappa$ and $\tilde{\kappa}$ can be realized from each $x \neq 0$ : For $\kappa$ this is Theorem 5.2(i), and for $\tilde{\kappa}$ the statement follows from Theorem 4.12 and Theorem 5.2(ii). However, in general $\kappa^{*}$ cannot be realized from $x \notin C^{-}$, compare Example 3.6. Note that in this example we have only one chain control set $E=\mathbf{P}$, and thus the only spectral interval over a chain control set is in this case $I(\mathbf{P})=\left[\kappa^{*}, \kappa\right]$, $I(\mathbf{P})$ being defined in (4.15). Therefore we see that the intervals of Lyapunov exponents over chain control sets can be too big to characterize precisely the stabilization behavior of (5.1). (Section 6. in Colonius and Kliemann $\left(1990^{d}\right)$ contains an example, where $C \cap \overline{C^{-}} \neq \phi, E=\mathrm{P}$, and $\kappa^{*}$ can be realized only from $x \in C^{-}$.)

(iii) Theorem 5.2(iv) says, in particular, that there exist Lyapunov regular matrix functions, whose Lyapunov exponents are arbitrarily close to the extremal exponents $\kappa$ (or $\tilde{\kappa}, \kappa^{*}$ ). This means that small perturbations of these functions will have the same stability behavior, compare Hahn (1967), Theorem 65.3.

As a consequence of Theorem 5.2 we obtain the following result on stabilization and destabilization: 


\subsection{Corollary.}

(i) The system (5.1) is (exponentially) destabilizable via an open loop control in $\mathcal{U}$ from some $x \neq 0$ (and hence from all $x \neq 0$ ) iff $\kappa>0$.

(ii) The system (5.1) is (exponentially) stabilizable via an open loop control in $\mathcal{U}$ from all $x \neq 0$ iff $\tilde{\kappa}<0$, and from some $x \neq 0$ (and hence from all $x \in C^{-}$) iff $\kappa^{*}<0$.

(iii) The system (5.1) is (exponentially) stabilizable (or destabilizable) iff there exists $u \in \mathcal{U}$ with $A_{0}+\Sigma u_{i}(t) A_{i}$ Lyapunov regular such that $\dot{x}=\left(A_{0}+\Sigma u_{i}(t) A_{i}\right) x$ is exponentially stable (or unstable, respectively).

Note that this corollary applies immediately to the problem of (de-)stabilization of linear systems via bounded, time varying output feedback.

It remains to compute the quantities $\kappa, \tilde{\kappa}, \kappa^{*}$ from (5.5). In specific cases, this can be done explicitly (compare e.g. the linear oscillator with controlled restoring force in Section 6 of Colonius and Kliemann $\left(1990^{d}\right)$ ), but in general one needs numerical procedures to compute these numbers. The problem can actually be formulated as an optimal control problem with infinite time, average cost criterion (compare Colonius and Kliemann (1989)), for which algorithms are available.

5.5. REMARK: The problem of stabilization of bilinear (or, in general, of nonlinear) control systems can also be approached via high gain techniques, which require fast, unbounded controls. Results in this direction have been obtained e.g. by Meerkov (1980), Bellman et al. (1985, 1986), Knobloch (1988), Colonius and Kliemann (1990 ${ }^{d}$, Section 7), or by Arnold et al. (1983) and Arnold (1989) in a stochastic context.

The remainder of this section is devoted to another important topic in systems theory, namely robustness, which can be approached e.g. via Lyapunov exponents. Assume we are given a linear control system

$$
\dot{x}(t)=\left(A_{0}+A(t)\right) x+B u, \quad t \in \mathbf{R}, \quad x(0)=x_{0} \in \mathbf{R}^{d},
$$

where $A(t)$ represents the uncertainty about some (or all) parameters of the given systems matrix $A_{0}$. The problem is to find criteria, under which for all uncertainties the system is stable (for $B=0$ ) or stabilizable (e.g. via output feedback). We will restrict ourselves here to the stability question (for stabilization criteria using Lyapunov exponents see Colonius and Kliemann $\left.\left(1990^{f}\right)\right)$.

More precisely, define the uncertainty range $U_{\rho} \subset \mathrm{R}^{m}$ for $\rho \geq 0$ by

$$
U_{\rho}=\left\{u \in \mathbf{R}^{m} ;|u| \leq \rho\right\}
$$

where $|\cdot|$ denotes any norm in $\mathbf{R}^{m}$ (often the Euclidean norm or the interval norm $u_{i} \in\left[\rho \alpha_{i}, \rho \beta_{i}\right], \alpha_{i}<0<\beta_{i}$ for $i=1 \ldots m$, are used.) The uncertain system (without input) is then modeled as

$$
\dot{x}(t)=\left(A_{0}+\sum_{i=1}^{m} u_{i}(t) A_{i}\right) x=: A(u) x, \quad\left(u_{i}(t)\right) \in U_{\rho}
$$


If $A_{0}$ is a stable matrix (i.e. all real parts of its eigenvalues are negative), then the system (5.7) is stable for all uncertainties, if $\rho$ is small enough. The problem is to find the smallest $\rho$, such that for some uncertainty with values in $U_{\rho}(5.7)$ becomes unstable, which leads to the following definition:

Denote by $\mathcal{U}_{\rho}=\left\{u: \mathbf{R} \rightarrow U_{\rho}\right.$; locally integrable $\}$ the set of uncertainties of size $\rho$, and again by $\lambda(u, x)=\limsup _{t \rightarrow \infty} \frac{1}{t} \log |\varphi(t, x, u)|$ the Lyapunov exponents of the solutions of $\left(5.7_{\rho}\right)$.

5.6. Definition: Let $A_{0} \in g \ell(d, \mathbf{R})$ be a stable matrix. Then the (Lyapunov) stability radius of $A_{0}$ with respect to the uncertainty structure in $\left(5.7_{\rho}\right)$ is defined by

$$
\begin{aligned}
r_{L}\left(A_{0}\right) & =\inf \left\{\rho \geq 0 ; \sup _{x \neq 0} \sup _{u \in \mathcal{u}_{\rho}} \lambda(x, u) \geq 0\right\} \\
& =\inf \left\{\rho \geq 0 ; \kappa_{\rho} \geq 0\right\}
\end{aligned}
$$

where $\kappa_{\rho}$ is defined as in (5.5) with $\mathcal{U}_{\rho}$ as the set of admissible controls.

Similarly, instability radii can be defined using $\tilde{\kappa}$ and $\kappa^{*}$. In the literature one finds a wide variety of stability radii, e.g. for only constant uncertainties $u(t) \equiv u \in U_{\rho}$, or for $U_{\rho} \subset \mathrm{C}^{m}$, or for $\left\{U_{\rho} ; \rho \geq 0\right\}$ just an increasing family of subsets in $\mathbf{R}^{m}$, etc. (compare e.g. Hinrichsen and Pritchard (1990), or Colonius and Kliemann (1990 $)$ ). In this brief expose we will only be concerned with stability radii, and only with those that are defined through all time-varying uncertainties with values in $U_{\rho}$ given as above.

We will again assume a nondegeneracy condition on the vector fields of the projected system on $\mathbf{P}$, which in this context reads (with $h(u, s)$ defined as in (5.2)):

$$
\begin{aligned}
\operatorname{dim} \mathcal{L A}\left\{h(u, \cdot) ; u \in U_{\rho}\right\}(s)=d-1 & \text { for all } s \in \mathbf{P} \text { and } \\
& \text { some (and hence all) } \rho>0 .
\end{aligned}
$$

We first note some uniformity and smoothness properties of the stability radius $r_{L}$ :

For a function $u \in \mathcal{U}_{\rho}$ denote by $\Phi_{u}(t, s)$ the fundamental matrix of $\left(5.7_{\rho}\right)$, and define the Bohl exponent for this equation by

$$
k_{B}(u)=\limsup _{s, t-s \rightarrow \infty} \frac{1}{t-s} \log \left\|\phi_{u}(t, s)\right\| .
$$

This exponent indicates uniform asymptotic stability of a linear, time varying differential equation, while the largest Lyapunov exponent $k_{L}(u)$ indicates asymptotic stability, hence, in general, $k_{B}(u)>k_{L}(u)$. This is not true for the corresponding stability radii, compare Colonius and Kliemann $\left(1990^{e}\right)$, Theorem 5:

5.7. Proposition. $r_{L}(A)=r_{B}(A):=\inf \left\{\rho \geq 0 ; \sup _{u \in \mathcal{u}_{\rho}} k_{B}(u) \geq 0\right\}$.

Furthermore, the maximal Lyapunov exponent $k_{L}(u)$ need not be continuous, nor even semi continuous in $u \in U$ or $u \in \mathcal{U}$. However, under Assumption $\left(\mathrm{H}_{\rho}\right)$, this effect is smoothed out in the extremal exponents $\kappa_{\rho}$ for $\left\{U_{\rho}, \rho \geq 0\right\}$ as above: 
5.8. Proposition. The function $\rho \mapsto \kappa_{\rho}$ is continuous and increasing. In particular, the set $\left\{\rho \geq 0 ; \kappa_{\rho}=0\right\}$ is a closed, connected subset of $[0, \infty)$ and $r_{L}(A)=\inf \{\rho \geq$ $\left.0 ; \kappa_{\rho}=0\right\}$.

The result is proved in Colonius and Kliemann $\left(1990^{f}\right)$. Note that $\rho \mapsto \kappa_{\rho}$ can be constant on intervals in $[0, \infty)$, i.e. the function need not be strictly increasing.

The following result on the uniformity of $r_{L}(A)$ with respect to the initial value $x \neq 0$ is an immediate consequence of Theorem 5.2: Denote by $\kappa_{\rho}(x):=\sup _{u \in \mathcal{U}_{\rho}} \lambda(u, x)$ the maximal Lyapunov exponent of $\left(5.7_{\rho}\right)$, which can be realized from a point $x \neq 0$.

5.9. Proposition. For all $x \in \mathbf{R}^{d}, x \neq 0$ we have $r_{L}(A)=\inf \left\{\rho \geq 0 ; \kappa_{\rho}(x)=0\right\}$.

5.10. REMARK: Willems and Willems (1983) proved several results about the robustness of linear systems with respect to stochastic uncertainties. In our context, we have the following results, compare Colonius and Kliemann $\left(1990^{e}\right)$ for the precise set up. Let $M$ be a compact smooth manifold and $D_{\rho}: M \rightarrow g \ell(d, \mathbf{R})$ a family of smooth maps with $D_{\rho}[M]=U_{\rho}$. Denote by Stat the set of all stationary processes with values in $M$, and by Diff the stationary, ergodic, nondegenerate diffusion processes on $M$. For a process $\xi_{t}$ in Stat let $\varphi\left(t, x, \xi_{t}\right)$ be the solution of $\dot{x}=A\left(D_{\rho}\left(\xi_{t}\right)\right) x$ (compare $\left(5.7_{\rho}\right)$ for the definition of $A()$.$) , and define \lambda_{\rho}\left(\xi_{t}, x\right)=\limsup _{t \rightarrow \infty} \frac{1}{t} \log \left|\varphi\left(t, x, \xi_{t}\right)\right|$,

$$
\kappa_{\rho}\left(\xi_{t}\right)=\operatorname{ess} \sup \sup _{x \neq 0} \lambda_{\rho}\left(\xi_{t}, x\right)
$$

where the essential supremum is taken over the measure induced by $\xi_{t}$ in $\mathcal{U}$,

$$
g_{\rho}\left(\xi_{t}, p\right)=\sup _{x \neq 0} \limsup _{t \rightarrow \infty} \frac{1}{t} \log E\left(\left|\varphi\left(t, x, \xi_{t}\right)\right|^{p}\right) .
$$

Then we have for stationary processes in Stat

$$
\begin{aligned}
r_{L}(A) & =\inf \left\{\rho \geq 0 ; \sup _{\xi_{t} \in \text { Stat }} \kappa_{\rho}\left(\xi_{t}\right) \geq 0\right\} \\
& =\sup \left\{\rho \geq 0 ; \sup _{\xi_{t} \in \text { Stat }} g_{\rho}\left(\xi_{t}, p\right) \leq 0 \text { for all } 0<p<\infty\right\}
\end{aligned}
$$

and for processes $\xi_{t}$ in Diff:

$$
\begin{aligned}
r_{L}(A) & =\sup \left\{\rho \geq 0 ; g_{\rho}\left(\xi_{t}, p\right) \leq 0 \text { for all } 0<p<\infty\right\} \\
& \leq \inf \left\{\rho \geq 0 ; \sup _{\xi_{t} \in \text { Diff }} \kappa_{\rho}\left(\xi_{t}\right) \geq 0\right\},
\end{aligned}
$$

where we conjecture that the last inequality is actually an equality. (The connection of these results with large deviation theory is explained in Colonius and Kliemann $\left(1990^{e}\right)$.) These findings show that the Lyapunov stability radius of (5.7) (and therefore also the Bohl stability radius by Proposition 5.7) is also the stability radius for stochastic uncertainties, both in the pathwise and in the pth-moment sense. This is just one way, 
in which Lyapunov exponents for control systems and for stochastic systems lead to a unifying approach.

\section{A Nonlinear Example: The Controlled Verhulst Equation.}

In this section we will analyze a simple one-dimensional nonlinear example under the aspects discussed in Section 5. for linear and bilinear systems, where we will make use of the results in Sections 3. and 4.

Consider the controlled Verhulst equation in $\mathbf{R}^{1}$

$$
\dot{x}(t)=X_{0}(x(t))+u(t) X_{1}(t)=\alpha x(t)-x(t)^{2}+u(t) x(t),
$$

with $\alpha \in \mathbf{R}$ and $u(t) \in[A, B] \subset \mathbf{R}$. For $u \equiv 0$, this equation undergoes a transcritical bifurcation at $\alpha=0$, where the rest point $x^{0}=0$ changes from stable to unstable as $\alpha$ increases. We are here concerned with stabilization and robustness properties of (6.1), in particular in the vicinity of the bifurcation point.

6.1. REMARK: Equation (6.1) can be solved explicitly: In the half spaces $(-\infty, 0)$ and $(0, \infty)$ set $y=\frac{1}{x}$, which leads to the linear differential equation $\dot{y}=-(\alpha+u(t)) y+1$. Retransformation of the solution of this equation leads to

$$
\varphi(t, x, u)=\frac{\exp \left\{\alpha t+\int_{0}^{t} u(\tau) d \tau\right\}}{\frac{1}{x}+\int_{0}^{t} \exp \left\{\alpha s+\int_{0}^{s} u(\tau) d \tau\right\} d s}
$$

for all $u \in \mathcal{U}$ and all $x \neq 0$. From this expression, the asymptotic behavior of the solutions, including their Lyapunov exponents and possible finite explosion times can be computed. We will not use this solution (except for one statement in Remark 6.2.), but rather present a method that relies only on the zeros of the right hand side of (6.1) for constant $u \in U$, and which, therefore, can be used for any one-dimensional control system.

For one-dimensional systems it is convenient to picture the systems dynamics with rest points and signs of the right hand sides in the $U \times \mathbf{R}$ plane, which yields in the case 
of equation (6.1) the following 'bifurcation diagram':

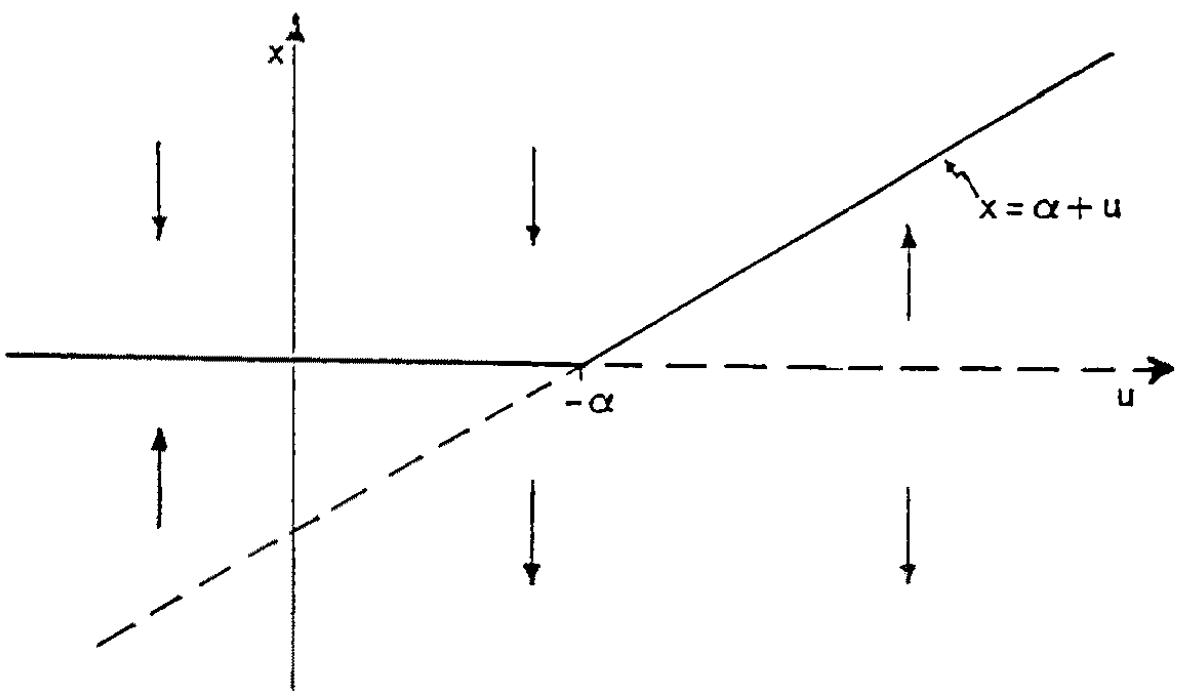

Here, for constant $u \in U$, the arrows indicate the direction of the vectorfields, the solid line corresponds to stable rest points, and the broken line to unstable ones. Define the corresponding two functions of zeros by

$$
\begin{aligned}
& z_{1}(u)= \begin{cases}\alpha+u & \text { for } u \leq-\alpha \\
0 & \text { for } u \geq-\alpha\end{cases} \\
& z_{2}(u)= \begin{cases}0 & \text { for } u \leq-\alpha \\
\alpha+u & \text { for } u \geq-\alpha .\end{cases}
\end{aligned}
$$

We start by analyzing the control sets, chain control sets, and their respective regions of attraction for the system (6.1). In Kliemann (1980) a general approach for finding the control sets of one-dimensional control systems was described, and extended in Colonius and Kliemann (1990') to chain control sets. (For this approach, Assumption (H) is not needed, and, in fact, it is violated here at the rest point $x^{0}=0$.) Using the results in Colonius and Kliemann $\left(1990^{c}\right)$, we obtain:

$$
\begin{aligned}
& D_{1}=\left(z_{1}(A), z_{1}(B)\right) \quad \text { if } A<-\alpha, \\
& D_{2}=\{0\}, \\
& D_{3}= \begin{cases}\left(z_{2}(A), z_{2}(B)\right] & \text { if } A \leq-\alpha, B>-\alpha, \\
{\left[z_{2}(A), z_{2}(B)\right]} & \text { if } A>-\alpha,\end{cases}
\end{aligned}
$$

and

$$
\begin{aligned}
& E_{1}=\overline{D_{1}} \text { if } B<-\alpha \\
& E_{2}= \begin{cases}D_{2} & \text { if } B<-\alpha \text { or } A>-\alpha \\
{\left[z_{1}(A), z_{2}(B)\right]} & \text { otherwise }\end{cases} \\
& E_{3}=D_{3} \text { if } A>-\alpha .
\end{aligned}
$$


The sets $D_{i}$ (and $E_{i}$ ) for $i=1, \ldots, 3$ are the possible control sets (and chain control sets, respectively) of (6.1).

The regions of attraction $\mathcal{A}\left(D_{i}\right)$ of the control sets (and similar for the chain control sets) are defined by

$$
\mathcal{A}\left(D_{i}\right)=\left\{(u, x) \in \mathcal{U} \times \mathbf{R} ; \pi_{\mathbf{R}} \omega(u, x) \subset D_{i}\right\} .
$$

Note that, in general, there may exist $(u, x) \in \mathcal{U} \times \mathbf{R}$, whose $\omega$-limit set intersects several control sets. These points are in no region of attraction, but they are a 'thin' set according to Lemma 4.2(vi). For chain control sets this cannot happen, compare Lemma 4.2(ii).

We have defined the regions of attraction of (chain) control sets as subsets of $\mathcal{U} \times \mathbf{R}$, and not of the state space $\mathbf{R}$ for reasons that will become clear from the following results for the system (6.1);

(i) If a control set is also a chain control set $E$, then there exists a compact neighborhood $N$ of $E$ such that for all $(u, x) \in \mathcal{U} \times N$ we have either $\omega(u, x) \subset \mathcal{E}$ or $\omega(u, x) \cap \mathcal{E}=\phi$, i.e. $\mathcal{E}$ is either an attractor or a repeller of the flow $(\mathcal{U} \times \mathbf{R}, \phi)$. (For the definition of $\mathcal{E}$ compare (3.7).)

(ii) If a control set $D$ is open and $E=\bar{D}$ is a chain control set, then there is a (compact) neighborhood $N$ of $E$ such that for all $(u, x) \in \mathcal{U} \times(N \backslash E)$ we have $\omega(u, x) \cap \mathcal{E}=\phi$, i.e. $\mathcal{E}$ is a repeller. In this case, we have the following control behavior in $D$ :

(a) For all $u \in \mathcal{U}$ there exist $x, y, z \in \bar{D}$ with

- $\phi(t, x, u) \in \bar{D}$ for all $t \geq 0$,

- $\lim _{t \rightarrow \infty} \varphi(t, y, u)=0 \notin \bar{D}$,

- $\lim _{t \rightarrow \infty} \varphi(t, z, u)=-\infty \notin \bar{D}$, and these are the only three possibilities for points in $\mathcal{U} \times \bar{D}$.

(b) For all $x \in D$ there exist $u, v, w \in \mathcal{U}$ such that

- $\pi_{\mathbf{R}} \omega(u, x) \subset D$,

- $\pi_{\mathbf{R}} \omega(v, x)=\{0\}$,

- $\pi_{\mathrm{R}} \omega(w, x)=\{-\infty\}$.

Note that, because of the continuity of the flow $\phi$, if $\pi_{\mathbf{R}} \omega(v, x)=\{0\}$ or $\pi_{\mathbf{R}} \omega(w, x)=\{-\infty\}$ then the same holds for open neighborhoods of $x$, and of $v$ or $w$.

In particular, (a) and (b) show that regions of attraction have to be defined on $\mathcal{U} \times \mathbf{R}$ and that these sets can have a very complicated structure.

(iii) It remains to discuss the invariant control sets $C$, which are not closed, and the variant control sets $D$ with $0 \in \bar{D}$. The closures of these sets are not chain control sets, and hence their lifts to $\mathcal{U} \times \mathbf{R}$ are neither attractors nor repellers. (Note that the corresponding chain control sets $E_{2}=\left[z_{1}(A), z_{2}(B)\right]$ are neither attractors nor repellers, too.) For the invariant control sets $C$ we have in this case:

(a) For all $x>0$ there exist $u, v, w \in \mathcal{U}$ such that - $\pi_{\mathbf{R}} \omega(u, x) \subset \operatorname{int} C$, 
- $\pi_{\mathbf{R}} \omega(v, x)=\{0\}$

- $\pi_{\mathbf{R}} \omega(w, x)=C \cup\{0\}$,

and there are $u \in \mathcal{U}$ such that for all $x>0$ one has $\omega(u, x)=\{0\}$.

Similarly, we obtain for the variant control sets $D$ : The possible limit behavior from $x \in D$ is the same as in (ii)(b), and there are $u \in \mathcal{U}$ such that for all $x>0$ one has $\omega(u, x)=\{-\infty\}$.

The proof of (i)-(iii) follows from the construction of (chain) control sets in Colonius and Kliemann $\left(1990^{c}\right)$, and Proposition 4.8. therein.

6.2. REMARK: Let $D$ be a control set of (6.1), then for all $\theta$-invariant (probability) measures $\rho$ on $\mathcal{U}$ there exists a $\phi$-invariant measure $\mu(d u, d x)=\mu_{u}(d x) \rho(d u)$ on $\mathcal{U} \times \mathbf{R}$ with supp $\mu \subset \mathcal{D}^{+}$, and all $\phi$-invariant probability measures have support in $\cup\left\{\mathcal{D}^{+} ; D\right.$ is a control set\}, compare Colonius and Kliemann $\left(1990^{\circ}\right)$, Theorem 4.3 and Corollary 4.9. In fact, it can be shown using the explicit solution in Remark 6.1, that for each $u \in \mathcal{U}$ there are exactly two ergodic invariant probability measures of the type $\mu_{u}$, compare Arnold and Boxler (1990). These findings are a starting point for a bifurcation theory of stochastic and control systems.

We now turn to the question of stabilization at the rest point $x^{0}=0$. This will be done via linearization around $x^{0}$, which together with the global control analysis above will give a complete picture in Proposition 6.3.

We have $\left.D X_{0}\right|_{x=0}=\alpha,\left.D X_{1}\right|_{x=0}=1$, and hence the linearized system at $x^{0}$ reads

$$
\dot{v}(t)=(\alpha+u(t)) v(t), \quad u(t) \in[A, B]
$$

Since the system (6.1) is one-dimensional, we do not need the projected system (2.6), but we can analyze (6.6) directly:

For constant $u \in[A, B]$ the linearized system is (exponentially) stable iff $u<-\alpha$, (then the Lyapunov exponent is $\lambda(u)=\alpha+u$ ), and (exponentially) unstable, iff $u>-\alpha$. For $u=\alpha$ we have $\lambda(u)=0$, a bifurcation takes place in (6.1) at this point, and $x^{0}=0$ is attractive in (6.1) for $x>0$. Because of the monotonicity of the vector field $X_{0}+u X_{1}$ in $u$, it suffices for the analysis of the stabilization behavior of the nonlinear system to consider constant controls. We obtain the following results:

\subsection{PROPOSITION.}

(i) The system (6.1) is (locally exponentially) stabilizable at $x^{0}=0$ iff $A<-\alpha$, the maximal rate of convergence is $\lambda(A)=\alpha+A$.

(ii) If (6.1) is stabilizable at $x^{0}=0$, then the maximal stabilization manifold (for $U=[A, B])$ is $M^{s}=\left(z_{1}(A),+\infty\right)$. For $x \notin M^{s}$ and all $u \in \mathcal{U}, u \neq A$ on a set of positive Lebesgue measure, we have $\varphi(t, x, u) \rightarrow-\infty$.

(iii) For $u=A$ Lebesgue almost everywhere, the system is stabilizable at $x^{0}=0$ from $x \in(0, \infty)$

(iv) The system is not exponentially stabilizable at $x^{0}=0$, iff $A \geq-\alpha$ (for all $x \neq 0$ ). 
The proof is a simple application of linearization theory with stable and unstable manifolds (for constant $u \in U$ ), together with the global results (i)-(iii) above. It is interesting to note that for $B>-\alpha$ there exists a second invariant control set, denoted by $D_{3}$ in (6.3), which also has a nonvoid region of attraction with points outside of $D_{3}$ by (iii) above. This rises the question, whether the system (6.1) can be stabilized e.g. at rest points or periodic solutions in $D_{3}$.

Finally, we discuss briefly some robustness properties of the nonlinear system (6.1). Consider the system

$$
\dot{x}(t)=\alpha x(t)-x(t)^{2}+u(t) x(t), \quad u(t) \in[\rho A, \rho B], \quad A<0<B, \quad \rho \geq 0,
$$

where now $u \in \mathcal{U}_{\rho}$ is interpreted as an uncertainty in the parameters of the vector field $X_{0}=\alpha x-x^{2}$, compare $(5.7 \rho)$ for the set up in the linear case. For the stable rest point $x^{0}=0$ of $X_{0}$ (i.e. $\left.\alpha<0\right)$ a local and a global stability radius can be defined: Denote by $\lambda(u), u \in \mathcal{U}$ again the Lyapunov exponents of the linearized system (6.6), and define the local stability radius by

$$
r_{L}\left(x^{0}\right)=\inf \left\{\rho \geq 0 ; \sup _{u \in \mathcal{U}_{\rho}} \lambda(u) \geq 0\right\}
$$

This radius corresponds to the existence of a stable manifold $M^{s}$ with $x^{0} \in \operatorname{int} M^{s}$, but it does not indicate, how large this stable manifold is, i.e. for which $x \in \mathbf{R}$ the long term behavior of $\varphi(t, x, u)$ is the same as that of $\varphi(t, x, u \equiv 0)$ with respect to the stability of $x^{0}$. We therefore define global stability radii for $x \in \mathbf{R}$ by

$$
r\left(x^{0} ; x\right)=\inf \left\{\rho \geq 0 ; \text { there exists } u \in \mathcal{U} \text { with } \pi_{\mathbf{R}} \omega(u, x) \neq\left\{x^{0}\right\}\right\} .
$$

Note that for linear systems the stability radius is always independent of $x \neq 0$ by Theorem 5.2(i), whereas the instability radii, based on $\tilde{\kappa}$ and $\kappa^{*}$, may depend on $x$, compare Colonius and Kliemann $\left(1990^{\circ}\right)$, Corollary 4 and Example 6.2, as well as Corollary 5.4. above.

From Proposition 6.3 we obtain immediately:

6.4. Proposition. Consider the nonlinear, uncertain system (6.7 $\rho$ ) with $\alpha<0$.

(i) $r_{L}\left(x^{0}\right)=-\frac{\alpha}{B}$

(ii) $r\left(x^{0} ; x\right)= \begin{cases}\frac{-\alpha}{B} & \text { for } x>0 \\ \frac{-\alpha+x}{B} & \text { for } x \in(\alpha, 0) \\ 0 & \text { for } x \leq \alpha .\end{cases}$

Similarly, local and global instability radii, i.e. the smallest $\rho$ such that $(6.7 \rho)$ is stabilizable for $\alpha>0$, can be analyzed. 


\section{REFERENCES}

Arnold, L. (1989). Stabilization by noise revisited. preprint, University of Bremen, FRG.

Arnold, L., P. Boxler (1989). Eigenvalues, bifurcation and center manifolds in the presence of noise. In: C. Dafermos (ed.) EQUADIFF '87, M. Dekker.

Arnold, L., P. Boxler (1990). Stochastic bifurcation: Instructive examples in dimension one. in: Wihstutz, V. (ed). Proceedings of the Conference on Stochastic Flows, Charlotte, NC, March 1990. Birkhäuser.

Arnold, L., H. Crauel, V. Wihstutz (1983). Stabilization of linear systems by noise. SIAM J. Control Optim. 21, 451-461.

Arnold, L., W. Kliemann, E. Oeljeklaus (1986). Lyapunov exponents of linear stochastic systems. In: Arnold, Wihstutz (1986), 85-125.

Arnold, L., V. Wihstutz (eds.) (1986). Lyapunov Exponents. Lecture Notes in Mathematics No. 1186, Springer.

Baxendale, P. (1986). The Lyapunov spectrum of a stochastic flow of diffeomorphisms. In: Arnold, Wihstutz (1986), 322-337.

Bellman, R., J. Bentsman, S. M. Meerkov (1985). On the vibrational siabilizability of nonlinear systems. J. Optim. Theory Appl. 46, 421-430.

Bellman, R., J. Bentsman, S. M. Meerkov (1986). Vibrational control of nonlinear systems: Vibrational stabilizability. IEEE Trans. Aut. Control AC-31, 710-716.

Byrnes, C., A. Isidori (1989). New results and examples in nonlinear feedback stabilization, Systems and Control Leters 12, 437-442.

Carverhill, A. (1985). Flows of stochastic dynamical systems: ergodic theory. Stochastics 14, 273-317.

Cesari, L. (1971). Asymptotic Behavior and Stability Problems in Ordinary Differential Equations. Springer, 3rd ed.

Colonius, F., W. Kliemann (1989). Infinite time optimal control and periodicity. Appl. Math. Opt. 20, 113-130.

Colonius, F., W. Kliemann $\left(1990^{a}\right)$. Some aspects of control systems as dynamical systems. Submitted. 
Colonius, F., W. Kliemann $\left(1990^{b}\right)$. Linear control semigroups acting on projective space. Submitted.

Colonius, F., W. Kliemann $\left(1990^{c}\right)$. Remarks on ergodic theory for stochastic and control flows. in: Wihstutz, V. (ed). Proceedings of the Conference on Stochastic Flows, Charlotte, NC, March 1990. Birkhäuser.

Colonius, F., W. Kliemann $\left(1990^{d}\right)$. Maximal and minimal Lyapunov exponents of bilinear control systems. To appear in J. Diff. Equations.

Colonius, F., W. Kliemann $\left(1990^{e}\right)$. Stability radii and Lyapunov exponents. In: Control of Uncertain Systems (D. Hinrichsen, B. Martensson, eds.), Birkhäuser, $19-55$.

Colonius, F., W. Kliemann $\left(1990^{f}\right)$. Stabilization of uncertain linear systems. To appear in: Modeling and Control of Uncertain Systems (G. DiMasi, A. Gombani, A. Kurzhanski, eds.), Birkhäuser.

Colonius, F., W. Kliemann $\left(1991^{a}\right)$. Limit behavior and control sets of nonlinear control systems. In preparation.

Colonius, F., W. Kliemann $\left(1991^{b}\right)$. The Lyapunov spectrum of bilinear control systems. In preparation.

Crauel, H. (1986). Lyapunov exponents and invariant measures of stochastic systems on manifolds. In: L. Arnold, V. Wihstutz (eds.).

Hahn, W. (1967). Stability of Motion. Springer.

Hinrichsen, D., A. J. Pritchard (1990). Destabilization by output feedback. preprint, University of Bremen, FRG.

Isidori, A. (1989). Nonlinear Control Theory. Springer. 2nd ed.

Johnson, R. A., K. J. Palmer, G. R. Sell (1987). Ergodic properties of linear dynamical systems. SIAM J. Math. Anal. 18, 1-33.

Kliemann, W. (1980). Qualitative Theory of Nonlinear Stochastic Systems (in German). Ph. D. dissertation. Bremen, FRG.

Knobloch, H. W. (1988). Stabilization of control systems by means of high gain feedback. In: Feichtinger, G. (ed). Optimal Control Theory and Economic Analysis 3. North Holland, 153-173. 
Lyapunov, M. A. (1893). General problems in the stability of motion (in Russian). Comm. Soc. Math. Kharkow. (reprinted in Ann. Math. Studies 17, Princeton (1949)).

Mañé, R. (1987). Ergodic Theory and Differentiable Dynamics. Springer.

Meerkov, S. M. (1980). Principle of vibrational control: Theory and applications. IEEE Trans. Aut. Control AC-25, 755-762.

Nemytskii, V. V., V. V. Stepanov (1960). Qualitative Theory of Differential Equations. Princeton University Press. (Russian edition 1949).

Nijmeijer, H., A. J. van der Schaft (1990). Nonlinear Dynamical Control Systems. Springer.

Oseledeč, V. I. (1968). A multiplicative ergodic theorem. Lyapunov characteristic numbers for dynamical systems. Trans. Moscow Math. Soc. 19, 197-231.

Ruelle, D. (1979). Ergodic theory of differentiable dynamical systems. Publ. I.H.E.S. 50, 275-320.

Sacker, R. J., G. R. Sell (1978). A spectral theory for linear differential systems. J. Differential Equations 37, 320-358.

San Martin, L. A. B. (1986). Invariant Control Sets on Fibre Bundles. Ph.D. dissertation, Warwick, England.

San Martin, L. A. B., L. Arnold (1986). A control problem related to the Lyapunov spectrum of stochastic flows. Matematica Aplicada e Computacional 5, $31-64$.

Sontag, E. D. (1983). A Lyapunov-like characterization of asymptotic controllability. SIAM J. Control Optim. 21, 462-471.

Sontag, E. D. (1989). Feedback stabilization of nonlinear systems. In: Kaashoek, M.A., J.H. van Schuppen, A.C.M. Ran (eds.), Robust Control of Linear Systems and Nonlinear Control, Proceedings of the International Symposium MTNS-89, Vol. II, Birkhäuser.

Sussmann, H. J. (1979) Subanalytic sets and feedback control. J. Differential Equations 31, 31-52.

Willems, J. L., J. C. Willems (1983). Robust stabilization of uncertain systems. SIAM J. Control Optim. 21, 352-374. 
Wonham, W. M. (1979). Linear Multivariable Control: A Geometric Approach. Springer. 